\title{
Essential role of hyperacetylated microtubules in innate immunity escape orchestrated by the EBV-encoded BHRF1 protein
}

Damien Glon ${ }^{1}$, Géraldine Vilmen ${ }^{1,2+}$, Daniel Perdiz ${ }^{3+}$, Eva Hernandez ${ }^{1}$, Guillaume Beauclair $^{1}$, Vincent Maréchal $^{2}$, Christian Poüs ${ }^{3,4}$, Marion Lussignol ${ }^{1 \#}$ and Audrey Esclatine ${ }^{1 \# *}$

1. Université Paris-Saclay, CEA, CNRS, Institute for Integrative Biology of the Cell (I2BC), 91198, Gif-surYvette, France

2. CRSA, Centre de Recherche Saint-Antoine, UMRS 938, INSERM, Sorbonne Université, 75012 Paris, France

3. INSERM UMR-S 1193, Université Paris-Saclay, 92296 Châtenay-Malabry, France

4. Biochimie-Hormonologie, APHP, Hôpitaux Universitaires Paris-Saclay, Site Antoine Béclère, 92141

Clamart, France

${ }^{\dagger}$ These authors contributed equally to this work.

\# These authors share senior authorship.

* For correspondence: audrey.esclatine@universite-paris-saclay.fr (AE)

Competing interests: The authors declare that no competing interests exist. 


\section{Abstract}

Innate immunity constitutes the first line of defense against viruses, in which mitochondria play an important role in the induction of the interferon (IFN) response. BHRF1, a multifunctional viral protein expressed during Epstein-Barr virus reactivation, modulates mitochondrial dynamics and disrupts the IFN signaling pathway. Mitochondria are mobile organelles that move through the cytoplasm thanks to the cytoskeleton and in particular the microtubule (MT) network. MTs undergo various posttranslational modifications, among them tubulin acetylation. In this study, we demonstrated that BHRF1 induces MT hyperacetylation to escape innate immunity. Indeed, expression of BHRF1 induces the aggregation of shortened mitochondria next to the nucleus. This mito-aggresome is organized around the centrosome and its formation is MT-dependent. We also observed that the BHRF1-induced hyperacetylation of MTs involves the $\alpha$-tubulin acetyltransferase ATAT1. Thanks to a non-acetylatable $\alpha$-tubulin mutant, we demonstrated that this hyperacetylation is necessary for the mito-aggresome formation. We investigated the mechanism leading to the clustering of mitochondria, and we identified dyneins as motors that are required for the mitochondrial aggregation. Finally, we demonstrated that BHRF1 needs MT hyperacetylation to block the induction of the IFN response. Indeed, in the absence of MT hyperacetylation, BHRF1 is unable to initiate the sequestration of mitochondria into autophagosomes, and mitophagy, which is essential to inhibiting the signaling pathway. Therefore, our results reveal the role of the MT network, and its acetylation level, in the induction of a pro-viral mitophagy. 


\section{Introduction}

The innate immunity system provides the first line of defense against different invading pathogens. This process is based on the sensing of motifs from the foreign organism, called the pathogenassociated molecular pattern (PAMP), by different host pattern recognition receptors (PRR). In the case of a viral infection, their recognitions notably lead to the induction of the interferon (IFN) response, which leads to the expression of interferon-stimulated genes and the synthesis of cytokines known for their antiviral properties (Lee and Ashkar, 2018).

Mitochondria carry out a crucial role in many cellular processes ranging from energy production to programmed cell death, from calcium homeostasis to cell immunity. They constitute a platform for signaling pathways involved in innate immunity thanks to the MAVS (mitochondrial antiviral signaling protein) receptor, predominantly localized at the mitochondrial outer membrane surface (Arnoult et al., 2011; West et al., 2011). The functions of mitochondria depend on their morphology, which is in turn dependent upon mitochondrial dynamics (including fission, fusion, and motility). Indeed, mitochondria are dynamic organelles that continually switch between cycles of fusion and fission to regulate the network structure (Giacomello et al., 2020). Fission occurs at mitochondria-endoplasmic reticulum (ER) contact sites, which facilitate the recruitment of Drp1 (dynamin related protein 1), which multimerizes and in turn triggers the severing of mitochondria (Pagliuso et al., 2018). Fusion is regulated by both MFN1/2 (mitofusins 1 and 2) and OPA1 (optic atrophy type 1 ) located the outer and inner mitochondrial membrane, respectively. Moreover, mitochondria are motile because they are actively recruited to specific cellular locations to respond to their functions. In eukaryotic cells, the cytoskeleton and notably microtubules (MTs) play a critical role in the distribution of mitochondria throughout the cytoplasm by facilitating their transport to areas with high metabolic demands (Frederick and Shaw, 2007; Kruppa and Buss, 2021).

The MT cytoskeleton is a highly dynamic polymer of $\alpha$ and $\beta$-tubulin heterodimers, which is involved in a variety of cellular functions, such as supporting cell structures, maintaining cell polarity, but also allowing the transport of organelles. Moreover, it has been shown that the MT network of viral infected cells could be used for intracellular transport of viral particle/genomic material to the sites of replication or assembly (Zheng et al., 2017). A broad range of MT-associated proteins (MAPs) contribute to regulate MT dynamic behavior. MAPs include kinesins and dyneins, which both allow the positioning of organelles and the long-distance transport of vesicles along MT tracks. Kinesins generally transport their cargos toward the plus-end of MTs at the cell periphery. Conversely, dyneins transport their cargos to the minus-end of MTs in the cell center (Hancock, 2014). The relationship between MTs and mitochondrial transport is not completely understood. Mitochondrial movement involves 
mitochondrial adaptors such as small mitochondrial Rho GTPases Miro1/2 and TRAK1/2 (LópezDoménech et al., 2018). Indeed, Miro proteins can engage both kinesin and dynein to mediate the bidirectional movement of mitochondria along MT tracts (Fransson et al., 2006; Frederick and Shaw, 2007; Tang, 2018).

MT functions are regulated by numerous post-translational modifications such as acetylation, detyrosination, phosphorylation, methylation or polyglutamylation (Janke and Magiera, 2020). Among them the acetylation of $\alpha$-tubulin, which mainly occurs on the Lysine in position 40 (K40), has emerged as an important regulator of numerous cell functions (Perdiz et al., 2011). For example, $\alpha$-tubulin acetylation, which is often linked to MT stabilization, may modulate the binding of MAPs (Dompierre et al., 2007; Mackeh et al., 2014; Reed et al., 2006) and therefore may modulate organelle movement. Indeed, stable MTs are particularly involved in mitochondrial transport (Friedman et al., 2010), and it has been reported that acetylation of $\alpha$-tubulin mediates dynein-dependent transport of mitochondria along MTs (Misawa et al., 2013).

In this work, we aimed at studying the role of the MT network in the innate immunity escape induced by the Epstein-Barr virus (EBV). BHRF1 is a viral Bcl-2 homolog (Khanim et al., 1997), and our group recently unravelled its novel functions in innate immunity, related to its mitochondrial localization (Vilmen et al., 2020). We demonstrated that BHRF1 disturbs mitochondrial dynamics and subsequently stimulates autophagy. More precisely, BHRF1 induces mitophagy, a cellular process that can specifically sequester and degrade mitochondria by autophagy, and we have shown that BHRF1induced mitophagy participates in the inhibition of type I IFN response by EBV (Vilmen et al., 2020). Here, we described the importance of the MT hyperacetylation on the mitochondrial clustering and, more unexpectedly, on the capability of BHRF1 to counteract the IFN induction. 
Results

\section{BHRF1-induced mito-aggresomes are organized around the centrosomes}

The EBV-encoded BHRF1 localizes to mitochondria, and its expression in HeLa cells dramatically alters mitochondrial dynamics and distribution, (Vilmen et al., 2020). By confocal microscopy, we observed abnormal mitochondrial aggregates close to the nucleus in BHRF1-expressing cells, whereas in control cells (empty vector [EV] transfection), mitochondria remained distributed throughout the cytoplasm (Figure 1A). This mitochondrial phenotype, known as mito-aggresome, is in accordance with our previous observations. We arbitrarily consider that, when the compaction index $(\mathrm{Cl})$ of mitochondria is above 0.4 , the cell presents a mito-aggresome, whereas when the $\mathrm{Cl}$ value is below 0.4 , mitochondria are distributed homogeneously throughout the cell cytoplasm (Narendra et al., 2010; Vilmen et al., 2020). The expression of BHRF1 significantly increased the $\mathrm{Cl}$ values, which corresponds to the formation of a mito-aggresome in almost $80 \%$ of the cells (Figure 1B). Since BHRF1 is associated with mitochondrial fragmentation (Figure $1 A$ - insets), we precisely evaluated mitochondrial shape by calculation of the aspect ratio (AR) and the form factor (FF), two parameters which reflect the mitochondrial length and the branching of mitochondria, respectively. As calculated in Figure $1 \mathrm{C}$, BHRF1 expression significantly reduced both AR and FF parameters, confirming an induction of mitochondrial fission.

As mitochondria localize near the nucleus, the involvement of the MT network in this mitochondrial redistribution was investigated. In mammalian cells, mitochondria move along cytoskeletal tracks to sites of high-energy demand in an MT-dependent manner (Detmer and Chan, 2007). At interphase, the MT network radiates from the centrosome, the main MT organizing center for directing the polarity and the orientation of MTs. By co-staining BHRF1-expressing cells for mitochondria and pericentrin, a classical centrosomal marker, we observed that mitochondria are concentrated around the centrosome to form a mito-aggresome (Figure 1D). Moreover, a 3D reconstruction confirmed the clustering of BHRF1 in the vicinity of the nucleus around the centrosome (Supplementary file 1).

\section{The MT network is required for BHRF1-induced mito-aggresome formation}

To test whether MTs play a role in the mitochondrial redistribution following BHRF1 expression, the whole MT network was disassembled using a treatment mixing nocodazole, a classical MT-destabilizing drug, and exposure to cold (Geeraert et al., 2010). As shown in Figure $1 E$ - left panel this treatment disassembled MTs (fuzzy staining of $\alpha$-tubulin). The removal of nocodazole followed by 1 hour in a warm complete medium at $37^{\circ} \mathrm{C}$ restored the MT network (Figure $1 \mathrm{E}$ - right panel). In cells without BHRF1 expression, mitochondria were homogeneously distributed in the cytoplasm, and the disorganization of the MT network had no impact on their distribution. Conversely, mitochondrial 
aggregation in BHRF1-expressing cells was abolished when MTs were depolymerized (Figure 1E - left panel) and mitochondria were then distributed throughout the cell ( $\mathrm{Cl}$ values mainly below 0.4 ). This phenomenon was reversible since the mitochondria rapidly re-aggregated to different extents within 1 hour after reassembly of the MT network (most of the $\mathrm{Cl}$ values above 0.4) (Figure 1E - right panel). In contrast, mitochondria in non-transfected neighboring cells remained scattered within the cytoplasm after nocodazole removal. The quantification of mito-aggresomes in BHRF1-positive cells showed that whereas less than $20 \%$ of the cells presented mitochondrial aggregation after depolymerization treatment (Figure 1F), this proportion went back to more than $50 \%$ after repolymerization of the MTs (compare to $80 \%$ without treatment). We concluded that the MT network is required for the mito-aggresome formation.

As the actin network is also known to be involved in mitochondrial transport and fission (De Vos et al., 2005; López-Doménech et al., 2018), we also checked if F-actin disorganization affects the mitochondrial phenotype induced by BHRF1. In order to do so, we treated HeLa cells with cytochalasin $B$ to disrupt F-actin but we did not observe any changes in the mitochondrial phenotype in BHRF1positive cells (Supplementary file 2A). BHRF1 still induced the mitochondrial aggregation and fission at the same level as in the control (Supplementary file 2B and C). Therefore, the actin network does not seem to be involved in the mitochondrial phenotype.

\section{BHRF1 induces MT hyperacetylation, which is required for the mito-aggresome formation}

We next explored whether BHRF1 could modify the MT network by assessing its acetylation level. Upon BHRF1 expression, whereas $\alpha$-tubulin staining was unchanged, acetyl- $\alpha$-tubulin was redistributed and colocalized with BHRF1 (Figure 2A). Moreover, we observed a brighter signal of acetyl- $\alpha$-tubulin, suggesting an increase in $\alpha$-tubulin acetylation. This was confirmed by western-blot analysis, showing an increase of $60 \%$ under BHRF1 expression (Figure $2 B$ ).

To determine whether this hyperacetylation could be involved in BHRF1-induced mitochondrial alterations, we expressed a non-acetylatable $\alpha$-tubulin mutant (mCherry- $\alpha$-tubulin K40A) which prevents MT hyperacetylation (Dompierre et al., 2007). It has previously been reported that this nonacetylatable $\alpha$-tubulin mutant does not alter the MT network and is present in the entire MT network (Geeraert et al., 2010). Notably, when $\alpha$-tubulin K40A is expressed, endogenous $\alpha$-tubulin still remains acetylatable. However, the MT network's ability to be hyperacetylated is prevented, since $\alpha$-tubulin acetylation only occurs on protofilaments and the MT network is totally decorated with this nonacetylatable $\alpha$-tubulin. We thus verified by immunoblot analysis that BHRF1 was unable to induce hyperacetylation of $\alpha$-tubulin when the mutant $\alpha$-tubulin K40A was expressed (Figure $2 C$ ). Then, the impact of $\alpha$-tubulin K40A on the clustering of mitochondria was analyzed (Figures 2D and E). In cells 
co-expressing $\alpha$-tubulin K40A and BHRF1, $\mathrm{Cl}$ mean value was below 0.4 and mitochondria were homogeneously distributed throughout the cytoplasm, similarly to control cells. Thus, we concluded that BHRF1-induced mito-aggresome formation depends on MT hyperacetylation.

Interestingly, we observed that without $\alpha$-tubulin hyperacetylation, BHRF1 was still able to promote mitochondrial fragmentation (Figure 2F). Confocal images (Figure 2D, insets) clearly showed that upon BHRF1 expression (for both WT and K40A conditions) mitochondria were drastically smaller. Therefore, hyperacetylation of MTs is essential for BHRF1-induced mito-aggresome formation but not for mitochondrial fission.

\section{MT hyperacetylation is necessary to the inhibition of type I IFN response by BHRF1}

Mitochondria appear to be central organelles for the induction of antiviral innate immunity. RIG-I (retinoic acid-inducible gene) and MDA5 (melanoma differentiation-associated protein 5) are two cytoplasmic PRRs that detect viral genome. Upon activation, these receptors are translocated to the mitochondria to interact with the mitochondrial-resident protein MAVS that recruits and activates TBK1 (TANK-binding kinase 1). This kinase is required for the phosphorylation of the transcription factors IRF3 and IRF7 (interferon regulatory factors 3 and 7), leading to their dimerization, nuclear translocation and the subsequent activation of type I IFN promoter (Arnoult et al., 2011; West et al., 2011). Our group and others previously demonstrated that impairing the mitochondrial morphodynamics leads to the inhibition of IFN response (Castanier et al., 2010; Chatel-Chaix et al., 2016; Vilmen et al., 2020; Yoshizumi et al., 2014). Considering the importance of tubulin hyperacetylation for the formation of mito-aggresomes, we sought to know whether MT hyperacetylation was required for the inhibition of type I IFN response by BHRF1. We performed an IFN- $\beta$ promoter-driven luciferase reporter assay in BHRF1-expressing HEK293T cells (Figure 3A). To activate the IFN-signaling pathway, we expressed an active form of RIG-I, designated $\triangle R I G-I$ (2xCARD) (Yoneyama et al., 2004), which constitutively activates MAVS-dependent pathway through CARD domains interaction. As previously observed, BHRF1 dramatically blocked the IFN- $\beta$ promoter activation, however, the expression of the $\alpha$-tubulin K40A significantly prevented this inhibition (Figure 3A). To confirm our results, we examined the cellular localization of IRF3 by immunofluorescence. In basal conditions, IRF3 staining is diffuse throughout the cytoplasm. When $\triangle$ RIG-I (2XCARD) is expressed, IRF3 translocates from the cytoplasm into the nucleus but expression of BHRF1 blocked this translocation (Figure 3B). However, when MT hyperacetylation was prevented, BHRF1 was not able to block IRF3 nuclear translocation (Figure 3B). Quantification of this assay showed that expression of the non-acetylatable form of $\alpha$-tubulin totally restored IRF3 nuclear translocation upon BHRF1 expression (Figure 3C). Therefore, our results demonstrated that MT hyperacetylation is required for BHRF1 to inhibit type I IFN response. 


\section{BHRF1 requires acetyltransferase ATAT1 to modify MTs and form mito-aggresomes.}

Thereafter, we wanted to characterize the mechanism of MT hyperacetylation induction by BHRF1. Tubulin acetylation results from the balance between the activities of tubulin acetyltransferases and deacetylases. Therefore, a hyperacetylation of the MTs can be the consequence of either the inhibition of deacetylases or the activation of an acetyltransferase. First of all, we wanted to investigate a potential inhibitory role of BHRF1 on the two main $\alpha$-tubulin deacetylases, HDAC6 (histone deacetylase 6) and SIRT2 (sirtuin type 2) (Hubbert et al., 2002; North et al., 2003). Indeed, it has been observed that knockdown of SIRT2 results in an aberrant mitochondrial distribution in the perinuclear region (Misawa et al., 2013), and another study showed that the depletion of SIRT2 results in nuclear envelope shape defects (Kaufmann et al., 2016). These observations are clearly similar to the effect of BHRF1 on mitochondria, as well as its distribution around the nuclear envelope and the modification of the nucleus shape (Figure 1A). Thus, we performed an in vitro tubulin deacetylation assay (Mackeh et al., 2014). This test consists of mixing the highly acetylated porcine brain tubulin with lysates of BHRF1-expressing cells. Lysate of control EV-transfected cells reduces the acetylation of tubulin by approximately $50 \%$ compared to condition without cell lysate (Figure $4 \mathrm{~A}$ ). The same deacetylase activity is observed with BHRF1-positive lysates, indicating that BHRF1 does not inhibit the deacetylases.

We subsequently investigated the role of ATAT1 (alpha-tubulin N-acetyltransferase 1) (Shida et al., 2010). We knocked down ATAT1 expression by a siRNA approach and observed no more detectable acetylation activity under BHRF1 expression (Figure 4B), indicating involvement of ATAT1 in BHRF1induced hyperacetylation. Then, we checked the mitochondrial network and observed that the knockdown of ATAT1 prevents the mito-aggresome formation but not mitochondrial fission (Figure 4C-E). These results led us to conclude that BHRF1 activates the acetyltransferase ATAT1, leading to MT hyperacetylation and subsequent mito-aggresome formation.

\section{Mito-aggresome formation requires retrograde transport of mitochondria via dyneins}

As observed in Figure 1E, after removal of nocodazole and regrowth of the MT network, mitochondria rapidly re-aggregated in BHRF1-expressing cells, indicating that an MT-dependent motor activity is involved in the mito-aggresome formation. Moreover, the hyperacetylation of MTs has been previously shown to facilitate the recruitment and binding of MAPs on the tubulin (Dompierre et al., 2007). We therefore hypothesized that BHRF1-induced mitochondrial aggregation next to the nucleus could use dyneins. To investigate this hypothesis, we inhibited dynein functions by overexpression of p50-dynamitin. The overexpression of one subunit of the dynactin complex (p50-dynamitin) disrupts it and results in the dissociation of dyneins from the MT network. We observed that the expression of 
this construction totally prevents mitochondrial aggregation in BHRF1-expressing cells (Figures 5A and $5 B)$. Moreover, the ability of BHRF1 to induce mitochondrial fission was decreased when dyneins were inhibited, as we observed a partial but significant restoration of mitochondrial fission parameters (AR and FF; Figure 5C).

To confirm the importance of dynein motors in the mito-aggresome formation, we treated cells with ciliobrevin-D, a specific inhibitor of dyneins, which blocks their gliding along MTs and their ATPase activity. As with overexpression of p50-dynamitin, BHRF1 was not able to induce mito-aggresomes in cells treated with ciliobrevin-D (Supplementary file 4B and 4C). However, BHRF1 still induced a slight mitochondrial fission phenomenon (Supplementary file $4 B$ and $4 D$ ). Altogether, these results demonstrate that fragmented mitochondria are aggregated next to the nucleus in a dynein-dependent manner.

MT hyperacetylation does not affect BHRF1-pro-autophagic activity, but is required for the first step of mitophagy induction

Our group previously demonstrated that BHRF1 first stimulates autophagy and then induces mitophagy, the selective removal of mitochondria (Vilmen et al., 2020). Autophagy is a conserved cellular process allowing the removal and recycling of damaged or supernumerary cellular components. This process involves double-membrane vesicles, called autophagosomes, which fuse with lysosomes to degrade the content. Selective autophagy consists of the recognition of a specific cargo by a molecular receptor that links the cargo to the autophagosome for its removal (Green and Levine, 2014).

Several studies demonstrated the role of MTs during the autophagic process, notably during the formation of the autophagosomes (Geeraert et al., 2010; Köchl et al., 2006; Mackeh et al., 2013). Thus, we investigated whether BHRF1 could stimulate autophagy dependent on MT hyperacetylation. The autophagic flux was studied in HeLa cells co-expressing $\alpha$-tubulin K40A and BHRF1. We first analyzed by immunofluorescence the accumulation of LC3 (microtubule associated protein 1 light chain 3), a classical marker of autophagosomes in the presence or absence of chloroquine (CQ), an inhibitor of the autophagic flux. When non-acetylatable $\alpha$-tubulin was expressed, BHRF1 induced a clear accumulation of LC3 dots in the cytoplasm, and even more after CQ treatment (Figure 6A). We quantified LC3 dots and we observed no difference when MTs were hyperacetylated or not (Figure 6B and supplementary file 5A). We confirmed the result by analyzing the accumulation of lipidated LC3 (LC3-II) by immunoblot and we observed an increase in LC3-II levels in BHRF1-expressing cells, independently of MT hyperacetylation (Figure $6 C$ and Supplementary file 5B). This increase is 
261 potentiated after CQ treatment. Therefore, we concluded that BHRF1 stimulates the biogenesis of 262 autophagosomes and the autophagic flux independently of MT hyperacetylation.

263 Mitochondrial aggregation in the perinuclear region has been already documented as essential to the 264 efficient removal of mitochondria by mitophagy (Lee et al., 2010). Although autophagy stimulation 265 occurs independently of MT hyperacetylation, we investigated whether BHRF1-induced mitophagy 266 depends on tubulin hyperacetylation by evaluating the colocalization between mitochondria and 267 autophagosomes in cells co-expressing $\alpha$-tubulin K40A and BHRF1. When MT hyperacetylation was 268 prevented, mitochondria did not colocalize anymore with autophagosomes (Figure 6D). The 269 quantification of colocalization intensity, measured by Manders' coefficient, confirmed that 270 mitochondrial sequestration in autophagic vesicles did not occur when the mito-aggresome formation 271 was impeded (Figure 6E).

272 We then decided to investigate whether, conversely, autophagy was involved in BHRF1-induced MT 273 hyperacetylation. We observed that inhibition of autophagy by treatment with 3-methyladenine (3274 MA) or spautin-1 impeded the MT hyperacetylation (Supplementary file 5C).

275 Altogether, these results demonstrated that MT hyperacetylation was essential for the mitochondrial 276 sequestration but not for the induction of autophagy. In fact, BHRF1 needs autophagy activation to 277 induce MT hyperacetylation. Therefore, we uncovered a novel mechanism to counteract anti-viral 278 immunity, involving the initiation of mitophagy through the induction of tubulin hyperacetylation by 279 BHRF1. 
We previously demonstrated that EBV-encoded BHRF1 blocks the IFN response by modifying the mitochondrial dynamics and by stimulating autophagy (Vilmen et al., 2020). Expression of this viral protein induces mitochondrial fission, followed by the relocalization of fragmented mitochondria close to the nucleus in the proximity of the centrosome (Figure 1 and Supplementary file 1). Mitochondria are usually driven over long distances along MTs by motor proteins, thanks to the Miro/TRAK adaptor complex (Kruppa and Buss, 2021). Here, we demonstrate that the MT network is hijacked by BHRF1 to ensure the mito-aggresome formation and to consequently block the antiviral innate immunity (Figures 1 and 3). We notably observed that disruption of the MT network impedes mitochondrial trafficking to the centrosome, suggesting a dynamic process. In accordance, our results indicate that BHRF1 requires the MT-based motor dynein, that typically move towards the minus-end of MT, to regroup mitochondria in the perinuclear region (Figure 5 and Supplementary file 4).

The cytoplasmic dynein complex contains a pair of identical heavy chains which comprises the MTbinding domain. Each heavy chain harbors a motor domain that belongs to the AAA+ superfamily. Additional subunits complete this complex, each of which functions as a dimer; these are the intermediate chain, light-intermediate chain and three types of light chain (TCTEX, DYNLL1 and Roadblock) (Janke and Magiera, 2020; Roberts et al., 2013). It has been reported that DYNLL1 can interact with BCL2L11/BIM, a proapoptotic BH3-only protein expressed at the mitochondrial outer membrane, and can regulate its proapoptotic activity (Puthalakath et al., 1999; Singh et al., 2019). Interestingly, it has been shown that BIM binds to BECN1, a key regulator of autophagosome formation, linking BECN1 to DYNLL1 and MTs, and leading to autophagy inhibition (Luo et al., 2012). We and others demonstrated that BHRF1 can stimulate autophagy through its interaction with BECN1 and block apoptosis through its interaction with BIM (Desbien et al., 2009; Kvansakul et al., 2010; Vilmen et al., 2020). Therefore, we hypothesize that BIM could connect mitochondria to MTs by interacting with BHRF1 and the MT-trafficking apparatus. The formation of this complex could explain the mitochondrial movement toward the centrosome and participate in autophagy regulation.

In eukaryotic cells, MTs are generally built from 13 chains of $\alpha-\beta$ tubulin dimers known as protofilaments. Properties and behaviors of MTs can be modulated by numerous post-translational modifications on both $\alpha$ and $\beta$ tubulin (Janke and Magiera, 2020; Janke and Montagnac, 2017). We observed that the expression of BHRF1 increases the acetylation of $\alpha$-tubulin on the lysine in position 40 (K40) and that acetylated MTs are required for the mito-aggresome formation (Figure 2). Acetylation of $\alpha$-tubulin subtly changes its structure and disrupts the interaction between adjacent $\alpha$ tubulins of neighboring protofilaments (Eshun-Wilson et al., 2019). The loss of this connection weakens 
interactions between protofilaments, which facilitates their sliding and decreases the flexural rigidity of MTs, making them more resistant to mechanical bending-induced breakage and disassembly (Portran et al., 2017).

The hyperacetylation of MTs can result from either activation of tubulin acetyltransferases or inhibition of deacetylases (Li and Yang, 2015). Knockdown of the acetyltransferase ATAT1 by siRNA impedes the acetylation of $\alpha$-tubulin and the mito-aggresome formation upon BHRF1 expression (Figure 4). Therefore, our results demonstrate that BHRF1 induces MT hyperacetylation by activating ATAT1. The expression of the non-acetylatable K40A mutant form of tubulin confirmed that tubulin acetylation, and not just ATAT1 activity, is required for the mito-aggresome formation and sequestration of mitochondria in autophagosomes (Figures 2 and 6). We also investigated a potential inhibitory role of BHRF1 on tubulin deacetylases. However, our results showed that BHRF1 does not impact the global tubulin deacetylase activity of BHRF1-expressing cells, suggesting that activities of HDAC6 and SIRT2, the two main $\alpha$-tubulin deacetylases, are not inhibited by BHRF1 (Hubbert et al., 2002; North et al., 2003).

Hyperacetylation of MTs in the presence of BHRF1 could directly allow the spatial arrangement of mitochondria to the centrosome but the precise role of acetylated MTs will need to be further investigated. Misawa et al. demonstrated that accumulation of acetylated $\alpha$-tubulin by knockdown of SIRT2 results in an aberrant mitochondrial distribution in the perinuclear region (Misawa et al., 2013), similar to what was observed with BHRF1. Moreover, acetylated $\alpha$-tubulin is more abundant in stable MTs, which are particularly implicated in mitochondrial transport (Friedman et al., 2010). Interestingly, it has been shown that MT hyperacetylation in vitro and in cells causes the recruitment of dynein and kinesin-1 to MTs, which notably stimulates anterograde and retrograde transport of organelles in neurons or epithelial cells (Dompierre et al., 2007; Geeraert et al., 2010). BHRF1-induced acetylation of $\alpha$-tubulin could thus increase dynein recruitment to MTs, facilitating the traffic of fragmented mitochondria to the centrosome.

It is interesting to note that MT hyperacetylation induced by BHRF1 is not involved in mitochondrial fission, since expression of the non-acetylatable $\alpha$-tubulin mutant K40A or the knockdown of ATAT1 have no impact on mitochondrial length or branching parameters (Figure $2 \mathrm{~F}$ and Figure $4 E$ ). We previously demonstrated that BHRF1 increases mitochondrial fragmentation in a Drp1-dependent manner (Vilmen et al., 2020). Moreover, BHRF1 increases the dephosphorylation of Drp1 in position Ser637, which is then recruited to mitochondria to allow mitochondrial fission (Vilmen et al., 2020). In our context, the lack of direct involvement of acetylated MTs on mitochondrial fission could seem quite surprising, since it has been previously shown that MT hyperacetylation triggered by acute 
hyperosmotic stress enhanced mitochondrial fission (Perdiz et al., 2017). However, in the context of this acute stress, mitochondrial fission is associated with an increased level of Ser616 phosphorylation of Drp1, a different post-translational modification from the one observed upon BHRF1 expression. Implication of hyperacetylated-MT in the mitochondrial fission process may thus differ according to the type of cellular stress.

BHRF1 is a multifunction protein (e.g. anti-apoptotic and pro-autophagic) localized at both the ER and the mitochondrial membranes. Since mitochondrial fission occurs at contact sites between ER and mitochondria, ER-localized BHRF1 could be involved in the ER remodeling into tubules which enwrap mitochondria and initiate the constriction of mitochondrial membranes before the recruitment of Drp1 (Friedman et al., 2011). Conversely, the impact of BHRF1 on MT acetylation and mitochondrial trafficking could be related to its mitochondrial membrane localization.

We observed that MT hyperacetylation is not necessary to induce autophagy upon BHRF1 expression, whereas inhibition of autophagy impedes MT acetylation (Figure 6 and Supplementary file 5). This could seem unexpected, since several studies have demonstrated the role of MT acetylation in autophagosome formation and maturation, and the autophagy flux is directly correlated to MT acetylation levels (Esteves et al., 2019; Geeraert et al., 2010; Xie et al., 2010). For example, initiation of starvation-induced autophagy requires MT acetylation to recruit dynein and kinesin-1 and to allow JNK (c-Jun N-terminal kinase) activation, leading to BECN1 release (Geeraert et al., 2010). Very recently, it has been shown that p27, a tumor suppressor which promotes autophagy in response to glucose starvation, stimulates MT acetylation by binding to and stabilizing ATAT1, to allow the delivery of autophagosomes in the proximity of the centrosome for efficient fusion with lysosomes (Nowosad et al., 2021). However, MTs is not involved in autophagosome formation in basal autophagy (Mackeh et al., 2013). Although BHRF1-induced MT hyperacetylation has no role in non-selective autophagy, we observed that it is essential for BHRF1 to induce mitophagy since, in the absence of $\alpha$-tubulin acetylation, BHRF1 does not induce mitochondrial sequestration into autophagosomes (Figure 6). This suggests that formation of the autophagosomal membranes around mitochondria requires acetylated MTs.

Numerous strategies are used by viruses to counteract or evade the innate immune response. We describe in this study a novel mechanism of a viral immune escape. Indeed, we report that MT hyperacetylation induced by BHRF1 can block IFN production, via mitophagy stimulation. Several proteins expressed by different viruses, such as human parainfluenza virus type 3 , influenza A virus 
to mitochondria, induces complete mitophagy which leads to MAVS degradation and subsequently impairs innate immune response (Wang et al., 2020). PB1-F2 is a non-structural IAV-encoded protein constitutively localized to the mitochondrial inner membrane space (Yoshizumi et al., 2014). At mitochondria, the interaction of PB1-F2 with LC3B and mitochondrial TUFM (Tu translation elongation factor) similarly leads to mitophagy induction, MAVS degradation and restrains type I IFN production (Wang et al., 2020). Whether these pro-viral mechanisms depend on the tubulin acetylation level needs to be explored.

The role of MT-dependent mitochondrial movement has been previously described to be involved in the NLRP3 (NOD-like receptor family pyrin domain containing 3 ) inflammasome, a complex that also contributes to antiviral innate immunity by the secretion of pro-inflammatory cytokines, such as interleukin-1 $\beta$ and 18 (Zhao and Zhao, 2020). Detection of a viral infection or a danger signal can trigger the formation of this inflammasome, which is comprises of NLRP3, the adaptor protein ASC (apoptosisassociated speck-like protein containing a CARD), and the effector protease caspase-1. Mitochondrial dynamics and movement are involved in inflammasome activation, to bring closer the fraction of ASC which resides on the mitochondria to NLRP3, located in the ER under resting conditions. It has been reported that acetylated MTs and dyneins are essential for the relocalization of mitochondria in the perinuclear region and the inflammasome complex formation (Misawa et al., 2013). However, several studies have also reported that the activation of mitophagy can preclude NLRP3 inflammasome formation by preventing the release of mitochondrial DNA and ROS (Mishra et al., 2021; Zhong et al., 2016). The viral protein PB1-F2 negatively regulates the NLRP3 inflammasome (Yoshizumi et al., 2014), and this could be related to its ability to stimulate mitophagy (Wang et al., 2020). The activity of BHRF1 on the inflammasome is currently unknown but EBV encodes an miRNA that targets the NLRP3 mRNA (Haneklaus et al., 2012).

To conclude, we demonstrated how BHRF1, which is expressed during the early stages of EBV infection and some latency programs associated with lymphomas (Fitzsimmons and Kelly, 2017), reduces innate immunity by manipulating the MT network. We propose a model (Figure 7) in which EBV exploits acetylated MTs to change mitochondrial morphodynamics, thereby draining fragmented mitochondria toward the centrosome, segregating them from IFN signaling pathways in mitophagosomes to block IFN production and create an optimal environment for virus replication. We previously showed that BHRF1 expression induces mitochondrial fission activation and subsequently autophagy stimulation (Figure 7). We report here that MT hyperacetylation is downstream of these events. Beyond EBV infection and latency, these findings identify for the first time a role for acetylated MTs in regulating IFN response and innate immunity against viruses. 


\section{Materials and methods}

414

415

416

417

418

419

420

421

422

423

424

425

426

427

428

429

430

431

432

\section{Antibodies}

Primary antibodies used in this study included anti- $\beta$-actin (Merck Millipore, MAB1501), anti-BHRF1 (Merck Millipore, MAB8188), anti-c-Myc (Sigma-Aldrich, M4439), anti-HA (Santa Cruz Biotechnology, sc-9082), anti-IRF3 (Novus Biologicals, SD2062), anti-LC3 (MBL International, PM036 and M151-3B), anti-pericentrin (Sigma-Aldrich, HPA016820), anti-TOM20 (BD Biosciences, 612278), anti- $\alpha$-tubulin (Sigma-Aldrich, T6199) and anti-acetyl- $\alpha$-tubulin (Sigma-Aldrich, T6793). Secondary antibodies used in this study included Alexa Fluor 488 goat anti-rabbit, anti-mouse (Jackson ImmunoResearch, 111-545003, 115-545-003), Alexa Fluor 555 goat anti-rabbit, anti-mouse (Life technology, A21428, A21424), Alexa Fluor 647 donkey anti-rabbit, anti-mouse (Life technology, A31573, A31571). Horseradish peroxidase (HRP)-labeled goat anti-mouse and anti-rabbit secondary antibodies were purchased from Jackson ImmunoResearch Laboratories (115-035-003, 111-035-003).

\section{Cell culture and transfection}

HeLa and HEK293T cells were cultured at $37^{\circ} \mathrm{C}$ under $5 \% \mathrm{CO}_{2}$ in Dulbecco's modified eagle medium (DMEM; Gibco, 41965-039) supplemented with 10\% fetal calf serum (FCS; Dominique Dutscher, S181B$500)$.

DNA plasmid transfections were performed using Fugene HD transfection reagent (Promega Corporation, E2311) according to the manufacturer's protocol. One day prior to transfection, cells were seeded in 24 -well plates and incubated at $37^{\circ} \mathrm{C}$. On the day of transfection, DNA plasmids were dispersed in opti-MEM (Gibco, 31985-047) and Fugene HD was added to the mix (ratio DNA:Fugene equals 1:3). After incubation for $10 \mathrm{~min}$ at room temperature (RT), the transfection mix was added to the cells. Then cells were incubated at $37^{\circ} \mathrm{C}$ and $6 \mathrm{~h}$ post-transfection, DMEM supplemented with $10 \%$ FCS was added. Depending on the expression plasmids, cells were fixed $24 \mathrm{~h}$ or $48 \mathrm{~h}$ post-transfection.

\section{Extinction of ATAT1 expression}

To deplete ATAT1 in HeLa cells, we performed transfection of siRNA directed against ATAT1 encoding mRNA (or non-relevant siRNA as control). We used ON-TARGETplus siRNAs from Dharmacon as a pool of four siRNAs: 5-GUAGCUAGGUCCCGAUAUA-3; 5-GAGUAUAGCUAGAUCCCUU-3; 5GGGAAACUCACCAGAA CGA-3; and 5-CUUGUGAGAUUGUCGAGAU-3. The transfections of siRNA were performed using Oligofectamine reagent (Thermo Fisher Scientific; 12.252.011) according to the manufacturer's protocol and were repeated at days 1, 2, and 3 at a final concentration of $100 \mathrm{nM}$. At the end of day 3, transfection with BHRF1-HA was also performed using Fugene HD transfection reagent as described above. Cells were then used at day 4 for immunoblotting or immunofluorescence analyses. 


\section{In vitro tubulin deacetylation assay}

Cell lysates of control cells or cells transfected with BHRF1-HA plasmids were prepared using $10 \mathrm{mM}$ Tris buffer $\mathrm{pH}$ 8.0, supplemented with $1 \%$ Triton, $1 \mathrm{mM} \mathrm{NAD}^{+}$, and the protease inhibitor cocktail. Lysate aliquots containing $20 \mu \mathrm{g}$ of proteins were mixed with $2 \mu \mathrm{g}$ of soluble acetylated tubulin dimers purified from porcine brain (Mackeh et al., 2014; Walker et al., 1988) and adjusted to $50 \mu \mathrm{L}$ with NAD+containing PEM buffer ( $80 \mathrm{mM}$ PIPES, $2 \mathrm{mM}$ EGTA, $1 \mathrm{mM} \mathrm{MgCl} 2$ ). After $1 \mathrm{~h}$ at $37^{\circ} \mathrm{C}$, reactions were stopped by adding 4 X SDS-PAGE sample buffer and immediately subjected to SDS-PAGE and Western blotting of acetylated and total $\alpha$-tubulin.

\section{Immunoblot analysis}

Cells were lysed in lysis buffer (65 mM Tris, pH 6.8, 4\% SDS, 1.5\% $\beta$-mercaptoethanol, and a cocktail of protease/phosphatase inhibitors) and kept at $100^{\circ} \mathrm{C}$ for $10 \mathrm{~min}$. Protein extracts were resolved on SDSPAGE (12.5\%) and electro-transferred onto a polyvinylidene difluoride membrane (Amersham, 10600002). After 1 h of incubation in blocking buffer (PBS, 0.1\% Tween20, and 5\% BSA or nonfat dry milk), the membranes were probed overnight at $4^{\circ} \mathrm{C}$ with primary antibodies. Horseradish peroxidaselabeled antibodies were used as secondary antibody, and revelation was performed using the $\mathrm{ECL}$ detection system according to the manufacturer's instructions (Merck Millipore, WBKLS0500). Scanning for quantification of protein levels was monitored using ImageJ software. An anti- $\beta$-actin antibody was used to ensure equal loadings and normalize quantification.

\section{Immunofluorescence analysis}

For all indirect immunofluorescence, cells were cultured on glass coverslips in 24-well plates. Cell monolayers were washed with PBS and cells were fixed with paraformaldehyde (PFA; 4\%) in PBS or methanol, depending on the antibodies. The cells were treated with PBS containing $\mathrm{NH}_{4} \mathrm{Cl}(50 \mathrm{mM})$ for 10 min, permeabilized using PBS, Triton X-100 (0.2\%) for 4 min, washed twice with PBS and then incubated for $1 \mathrm{~h}$ in PBS, gelatin (0.2\%) supplemented with FCS (5\%) for blocking. Then the cells were incubated for $1 \mathrm{~h}$ with the appropriate primary antibody diluted in PBS, gelatin (0.2\%) at RT. Cells were washed three times with PBS and then incubated with the appropriate secondary antibody diluted in PBS, gelatin (0.2\%) for $1 \mathrm{~h}$ at RT. After washing, the nuclei were counterstained with DAPI. Coverslips were mounted in Glycergel (Agilent Dako, C056330-2) and observed using a Nikon Eclipse 80i epifluorescence microscope (Nikon Instruments) or a Leica TCS SP8 X inverted confocal microscope (Leica, USA). Photographic images were resized, organized, and labeled using Fiji-ImageJ software or LAS AF Lite. Imaris software was also used to perform 3D reconstruction video.

A specific procedure was performed for the colocalization study between mitochondria and autophagosomes. Permeabilization and blocking were performed by incubating the cells for $30 \mathrm{~min}$ 
with PBS, saponin (0.075\%; Sigma-Aldrich, 84,510), BSA (1\%), and FCS (5\%). Antibodies were diluted in PBS, saponin $(0.075 \%)$, and BSA (1\%). In addition, between each antibody incubation, cells were washed twice in PBS, saponin $(0.075 \%)$, BSA $(1 \%)$, once with PBS, saponin $(0.037 \%)$, BSA $(0.5 \%)$ and twice in PBS. Other steps were the same as described above.

\section{Luciferase reporter assays to assess IFN- $\beta$ promoter activity}

HEK293T cells were cultured in 24-well plates and transfected for $24 \mathrm{~h}$ using Fugene HD, with plasmids encoding the IFN- $\beta$ luciferase reporter (firefly luciferase; $100 \mathrm{ng}$ ), pRL-TK (renilla luciferase; $25 \mathrm{ng}$ ), pDRIG-I (2xCARD) (encoding a positive dominant form of RIG-I; $150 \mathrm{ng}$ ), plasmids expressing BHRF1HA (or EV as control; $150 \mathrm{ng}$ ) and mCherry-Tub (WT or K40A; $150 \mathrm{ng}$ ). One day after transfection, cells were lysed and measurement of firefly and renilla luciferase activities was performed using the dualluciferase reporter assay system (Promega Corporation, E1910) according to the manufacturer's protocol. Relative expression levels were calculated by dividing the firefly luciferase values by those of renilla luciferase and normalized to the control condition (without BHRF1-HA expression).

\section{Microtubules depolymerization and repolymerization}

To depolymerize MTs, HeLa cells were seeded directly onto glass coverslips and incubated one day before being transfected to express BHRF1. Twenty-four hours after transfection, cells were treated with $10 \mu \mathrm{M}$ nocodazole (Sigma-Aldrich, M1404) for $2 \mathrm{~h}$ at $37^{\circ} \mathrm{C}$ then $1.5 \mathrm{~h}$ at $4^{\circ} \mathrm{C}$, to depolymerize the MT network. Cells were immediately PFA 4\% fixed and then methanol-permeabilized $(\mathrm{t}=0 \mathrm{~h}$ before repolymerization). MTs and mitochondria were visualized by immunostaining of $\alpha$-tubulin and TOM20, respectively. To allow reassembly of the MT network, nocodazole was removed by repeated washes with PBS, and cells were left $1 \mathrm{~h}$ in warm complete medium ( $\mathrm{t}=1 \mathrm{~h}$ after MTs regrowth). Cells were then immunostained for BHRF1, TOM20, and $\alpha$-tubulin.

\section{Mitochondrial phenotype assessment}

BHRF1-induced mito-aggresomes were scored by measuring the $\mathrm{Cl}$ of mitochondrial staining, as we previously described (Vilmen et al., 2020), based on Narendra et al. study (Narendra et al., 2010). We arbitrary determined that a cell presents a mito-aggresome when the $\mathrm{Cl}$ is above 0.4 . The percentage of cells presenting a mito-aggresome was determined by counting at least 20 random cells in each condition from three independent experiments.

Mitochondrial morphology was assessed by measuring $A R$ and FF, which are indicators of mitochondrial length and branching, respectively. These parameters were quantified as described before (Koopman et al., 2006). Briefly, on Fiji-ImageJ software, images of mitochondria were convolved using the matrix developed by Koopman, then a threshold was applied to isolate mitochondria from background. Then cell outline was drawn and mitochondrial phenotype was assessed by the 
implement analysis particle tool. The AR value is given by the software and corresponds to the ratio between the major and minor axis of the ellipsis equivalent to the mitochondria. The FF value is defined as $\left(P_{\text {mit }}{ }^{2}\right) /\left(4 \pi A_{\text {mit }}\right)$, where $P_{\text {mit }}$ is the length of mitochondrial outline and $A_{\text {mit }}$ is the area of mitochondria. The mitochondrial fission parameters were determined by analyzing at least 15 random cells in each condition from three independent experiments.

\section{Plasmids}

We previously described the BHRF1-HA expression vector (Vilmen et al., 2020), which was constructed from a pcDNA3.1 expression vector (EV) purchased from Invitrogen (V79020). The mCherry- $\alpha$-tubulin WT-K40K control vector was kindly provided by Dr. R. Y. Tsien (Department of Pharmacology, Howard Hughes Medical Center, University of San Diego, USA) (Shaner et al., 2004) and the mutated vector (mCherry- $\alpha$-tubulin K40A) was a kind gift from Dr. F. Saudou (Institut Curie, Orsay, France) (Dompierre et al., 2007). The plasmids Flag- $\triangle$ RIG-I (2xCARD) and pIFN- $\beta$-Luc were previously described respectively (Yoneyama et al., 2004, 1996) and kindly provided by Dr. C. Lagaudrière (I2BC, Gif-sur-Yvette, France). The plasmid pRL-TK was purchased from Promega Corporation (E2241). The p50-dynamitin construct was a gift from Dr. S. Etienne-Manneville (Institut Pasteur, Paris, France) (Etienne-Manneville and Hall, 2001).

\section{Reagents}

MitoTracker Red CMXRos was purchased from Life Technologies (M7512) and used at $100 \mathrm{nM}$ for 30 min before cell fixation to stain the mitochondrial network. Phalloidin-Tetramethylrhodamine B isothiocyanate was purchased from Sigma-Aldrich (P1951) and used at $20 \mu \mathrm{g} / \mathrm{mL}$ during $1 \mathrm{~h}$ to stain Factin. DAPI was purchased from Thermo Fisher Scientific (D1306) and used at $300 \mathrm{nM}$ to stain dsDNA (nuclei). Nocodazole was purchased from Sigma-Aldrich (M1404) and used at $10 \mu \mathrm{M}$ to disrupt the MT network. Ciliobrevin D was purchased from Sigma-Aldrich (250401) and used at $40 \mu \mathrm{M}$ overnight to inhibit cytosolic dyneins. Cytochalasin B was purchased from Sigma-Aldrich (C6762) and used at 10 $\mu \mathrm{g} / \mathrm{mL}$ for $30 \mathrm{~min}$ to disrupt F-actin. Autophagic flux was monitored by the addition of chloroquine (Sigma-Aldrich, C6628) at $50 \mu \mathrm{M}$ for 2 to $4 \mathrm{~h}$. To inhibit autophagy, we used two different ways: cells were treated either with 3-MA (Sigma-Aldrich, M9281) at $5 \mathrm{mM}$ for $8 \mathrm{~h}$ or by spautin-1 (Sigma-Aldrich, SML0440) at $20 \mu \mathrm{M}$ for $8 \mathrm{~h}$.

\section{Results quantification}

The number of endogenous LC3 dots per cell was determined using Fiji-ImageJ software. All images were taken with the same microscope settings to allow for comparison. Images were converted into 8-bit. Cells' outlines were drawn and nuclei cropped to conserve only the cytoplasm. A thresholding of images was performed to detect only LC3 vesicles. Note that the value of the threshold was conserved 


\section{Funding}

\begin{tabular}{lll} 
Funder & Grand reference number & Author \\
\hline CNRS, Univ Paris-Sud, DIM MALINF Région IDF & N/A & Géraldine Vilmen \\
\hline Agence Nationale de la Recherche & ANR-14-CE14-0022 & Audrey Esclatine \\
\hline $\begin{array}{l}\text { The authors declare that the funders had no role in study design, data collection or interpretation, } \\
\text { or the decision to submit the work for publication. }\end{array}$ & \\
\hline
\end{tabular}

\section{Author contributions}

568 DG, GV, DP, conception and design, investigation, formal analysis, writing original draft; EH, 569 investigation, formal analysis; GB, formal analysis, writing original draft, VM, formal analysis, funding acquisition, supervision; $\mathrm{CP}$, formal analysis, provided materials; $\mathrm{ML}$, conception and design, formal 
571 analysis, writing original draft, supervision; $A E$, conception and design, formal analysis, funding

572 acquisition, writing original draft, supervision.

573

574

575

576

577

578

579

580

581

582

583

584

585

586

587

588

589

590

591

592

\section{Authors ORCIDs}

Damien Glon : https://orcid.org/0000-0001-8405-8805

Guillaume Beauclair : https://orcid.org/0000-0001-5323-5987

Vincent Maréchal : https://orcid.org/0000-0003-2689-6266

Christian Poüs : https://orcid.org/0000-0002-2502-7854

Marion Lussignol : https://orcid.org/0000-0001-7586-2041

\section{Supplementary files}

- Supplementary file 1. BHRF1 localizes in proximity of the centrosome.

- Supplementary file 2. The actin network is not involved in the formation of BHRF1-induced mitoaggresomes.

- Supplementary file 3. Expression of the non-acetylatable $\alpha$-tubulin K40A does not impact mitochondrial network.

- Supplementary file 4. Dyneins inhibition prevents BHRF1-induced mito-aggresomes.

- Supplementary file 5. BHRF1 stimulates autophagy when $\alpha$-tubulin WT is expressed, and autophagy is required to MT hyperacetylation.

- Supplementary file 6. Full-length western-blots.

\section{Data availability}

All data generated or analyzed during this study are included in this published article and its supplementary information files.

\section{References}

Arnoult D, Soares F, Tattoli I, Girardin SE. 2011. Mitochondria in innate immunity. EMBO Rep 12:901-910. doi:10.1038/embor.2011.157

Castanier C, Garcin D, Vazquez A, Arnoult D. 2010. Mitochondrial dynamics regulate the RIG-I-like receptor antiviral pathway. EMBO Rep 11:133-138. doi:10.1038/embor.2009.258

Chatel-Chaix L, Cortese M, Romero-Brey I, Bender S, Neufeldt CJ, FischI W, Scaturro P, Schieber N, Schwab Y, Fischer B, Ruggieri A, Bartenschlager R. 2016. Dengue Virus Perturbs Mitochondrial Morphodynamics to Dampen Innate Immune Responses. Cell Host Microbe 20:342-356. doi:10.1016/j.chom.2016.07.008

De Vos KJ, Allan VJ, Grierson AJ, Sheetz MP. 2005. Mitochondrial Function and Actin Regulate Dynamin-Related Protein 1-Dependent Mitochondrial Fission. Curr Biol 15:678-683. doi:10.1016/i.cub.2005.02.064

Desbien AL, Kappler JW, Marrack P. 2009. The Epstein-Barr virus Bcl-2 homolog, BHRF1, blocks apoptosis by binding to a limited amount of Bim. Proc Natl Acad Sci U S A 106:5663-5668. doi:10.1073/pnas.0901036106 
Detmer SA, Chan DC. 2007. Functions and dysfunctions of mitochondrial dynamics. Nat Rev Mol Cell Biol 8:870-879. doi:10.1038/nrm2275

Ding B, Zhang L, Li Z, Zhong Y, Tang Q, Qin Y, Chen M. 2017. The Matrix Protein of Human Parainfluenza Virus Type 3 Induces Mitophagy that Suppresses Interferon Responses. Cell Host \& Microbe 21:538-547.e4. doi:10.1016/i.chom.2017.03.004

Dompierre JP, Godin JD, Charrin BC, Cordelieres FP, King SJ, Humbert S, Saudou F. 2007. Histone Deacetylase 6 Inhibition Compensates for the Transport Deficit in Huntington's Disease by Increasing Tubulin Acetylation. J Neurosci 27:3571-3583. doi:10.1523/JNEUROSCI.003707.2007

Eshun-Wilson L, Zhang R, Portran D, Nachury MV, Toso DB, Löhr T, Vendruscolo M, Bonomi M, Fraser JS, Nogales E. 2019. Effects of $\alpha$-tubulin acetylation on microtubule structure and stability. Proc Natl Acad Sci U S A 116:10366-10371. doi:10.1073/pnas.1900441116

Esteves AR, Palma AM, Gomes R, Santos D, Silva DF, Cardoso SM. 2019. Acetylation as a major determinant to microtubule-dependent autophagy: Relevance to Alzheimer's and Parkinson disease pathology. Biochim Biophys Acta, Post-Translational Modifications In Brain Health And Disease 1865:2008-2023. doi:10.1016/j.bbadis.2018.11.014

Etienne-Manneville S, Hall A. 2001. Integrin-mediated activation of Cdc42 controls cell polarity in migrating astrocytes through PKCzeta. Cell 106:489-498. doi:10.1016/s0092-8674(01)00471$\underline{8}$

Fitzsimmons L, Kelly G. 2017. EBV and Apoptosis: The Viral Master Regulator of Cell Fate? Viruses 9:339-373. doi:10.3390/v9110339

Fransson Å, Ruusala A, Aspenström P. 2006. The atypical Rho GTPases Miro-1 and Miro-2 have essential roles in mitochondrial trafficking. Biochem Biophys Res Commun 344:500-510. doi:10.1016/j.bbrc.2006.03.163

Frederick RL, Shaw JM. 2007. Moving Mitochondria: Establishing Distribution of an Essential Organelle. Traffic 8:1668-1675. doi:10.1111/j.1600-0854.2007.00644.x

Friedman JR, Lackner LL, West M, DiBenedetto JR, Nunnari J, Voeltz GK. 2011. ER Tubules Mark Sites of Mitochondrial Division. Science 334:358-362. doi:10.1126/science.1207385

Friedman JR, Webster BM, Mastronarde DN, Verhey KJ, Voeltz GK. 2010. ER sliding dynamics and ERmitochondrial contacts occur on acetylated microtubules. J Cell Biol 190:363-375. doi:10.1083/jcb.200911024

Geeraert C, Ratier A, Pfisterer SG, Perdiz D, Cantaloube I, Rouault A, Pattingre S, Proikas-Cezanne T, Codogno P, Poüs C. 2010. Starvation-induced Hyperacetylation of Tubulin Is Required for the Stimulation of Autophagy by Nutrient Deprivation. J Biol Chem 285:24184-24194. doi:10.1074/ibc.M109.091553

Giacomello M, Pyakurel A, Glytsou C, Scorrano L. 2020. The cell biology of mitochondrial membrane dynamics. Nat Rev Mol Cell Biol 21:204-224. doi:10.1038/s41580-020-0210-7

Green DR, Levine B. 2014. To Be or Not to Be? How Selective Autophagy and Cell Death Govern Cell Fate. Cell 157:65-75. doi:10.1016/i.cell.2014.02.049

Hancock WO. 2014. Bidirectional cargo transport: moving beyond tug of war. Nat Rev Mol Cell Biol 15:615-628. doi:10.1038/nrm3853

Haneklaus M, Gerlic M, Kurowska-Stolarska M, Rainey A-A, Pich D, McInnes IB, Hammerschmidt W, O'Neill LAJ, Masters SL. 2012. Cutting Edge: miR-223 and EBV miR-BART15 Regulate the NLRP3 Inflammasome and IL-1ß Production. J Immunol 189:3795-3799. doi: $10.4049 /$ jimmunol.1200312

Hubbert C, Guardiola A, Shao R, Kawaguchi Y, Ito A, Nixon A, Yoshida M, Wang X-F, Yao T-P. 2002. HDAC6 is a microtubule-associated deacetylase. Nature 417:455-458. doi:10.1038/417455a

Janke C, Magiera MM. 2020. The tubulin code and its role in controlling microtubule properties and functions. Nat Rev Mol Cell Biol 21:307-326. doi:10.1038/s41580-020-0214-3

Janke C, Montagnac G. 2017. Causes and Consequences of Microtubule Acetylation. Curr Biol 27:1287-1292. doi:10.1016/i.cub.2017.10.044 
Kaufmann T, Kukolj E, Brachner A, Beltzung E, Bruno M, Kostrhon S, Opravil S, Hudecz O, Mechtler K, Warren G, Slade D. 2016. SIRT2 regulates nuclear envelope reassembly through ANKLE2 deacetylation. J Cell Sci 129:4607-4621. doi:10.1242/jcs.192633

Khanim F, Mackett M, Young LS, Dawson J, Meseda CA, Dawson C. 1997. BHRF1, a viral homologue of the $\mathrm{Bcl}-2$ oncogene, is conserved at both the sequence and functional level in different Epstein-Barr virus isolates. J Gen Virol 78:2987-2999. doi:10.1099/0022-1317-78-11-2987

Köchl R, Hu XW, Chan EYW, Tooze SA. 2006. Microtubules Facilitate Autophagosome Formation and Fusion of Autophagosomes with Endosomes: Role of Microtubules in AV Formation. Traffic 7:129-145. doi:10.1111/j.1600-0854.2005.00368.x

Koopman WJH, Visch H-J, Smeitink JAM, Willems PHGM. 2006. Simultaneous quantitative measurement and automated analysis of mitochondrial morphology, mass, potential, and motility in living human skin fibroblasts. Cytometry Part A 69A:1-12. doi:10.1002/cyto.a.20198

Kruppa AJ, Buss F. 2021. Motor proteins at the mitochondria-cytoskeleton interface. J Cell Sci 134:113. doi:10.1242/ics.226084

Kvansakul M, Wei AH, Fletcher JI, Willis SN, Chen L, Roberts AW, Huang DCS, Colman PM. 2010. Structural Basis for Apoptosis Inhibition by Epstein-Barr Virus BHRF1. PLoS Pathog 6:e1001236. doi:10.1371/journal.ppat.1001236

Lee AJ, Ashkar AA. 2018. The Dual Nature of Type I and Type II Interferons. Front Immuno/ 9:20612070. doi:10.3389/fimmu.2018.02061

Lee J-Y, Nagano Y, Taylor JP, Lim KL, Yao T-P. 2010. Disease-causing mutations in Parkin impair mitochondrial ubiquitination, aggregation, and HDAC6-dependent mitophagy. J Cell Biol 189:671-679. doi:10.1083/jcb.201001039

Li L, Yang X-J. 2015. Tubulin acetylation: responsible enzymes, biological functions and human diseases. Cell Mol Life Sci 72:4237-4255. doi:10.1007/s00018-015-2000-5

López-Doménech G, Covill-Cooke C, Ivankovic D, Halff EF, Sheehan DF, Norkett R, Birsa N, Kittler JT. 2018. Miro proteins coordinate microtubule- and actin-dependent mitochondrial transport and distribution. EMBO J 37:321-336. doi:10.15252/embj.201696380

Luo S, Garcia-Arencibia M, Zhao R, Puri C, Toh PPC, Sadiq O, Rubinsztein DC. 2012. Bim inhibits autophagy by recruiting Beclin 1 to microtubules. Mol Cell 47:359-370. doi:10.1016/j.molcel.2012.05.040

Mackeh R, Lorin S, Ratier A, Mejdoubi-Charef N, Baillet A, Bruneel A, Hamaï A, Codogno P, Poüs C, Perdiz D. 2014. Reactive Oxygen Species, AMP-activated Protein Kinase, and the Transcription Cofactor p300 Regulate $\alpha$-Tubulin Acetyltransferase-1 ( $\alpha$ TAT-1/MEC-17)dependent Microtubule Hyperacetylation during Cell Stress. J Biol Chem 289:11816-11828. doi:10.1074/jbc.M113.507400

Mackeh R, Perdiz D, Lorin S, Codogno P, Pous C. 2013. Autophagy and microtubules - new story, old players. J Cell Sci 126:1071-80. doi:10.1242/ics.115626

Misawa T, Takahama M, Kozaki T, Lee H, Zou J, Saitoh T, Akira S. 2013. Microtubule-driven spatial arrangement of mitochondria promotes activation of the NLRP3 inflammasome. Nat Immunol 14:454-460. doi:10.1038/ni.2550

Mishra SR, Mahapatra KK, Behera BP, Patra S, Bhol CS, Panigrahi DP, Praharaj PP, Singh A, Patil S, Dhiman R, Bhutia SK. 2021. Mitochondrial dysfunction as a driver of NLRP3 inflammasome activation and its modulation through mitophagy for potential therapeutics. Int J Biochem Cell Biol 136:106013-106021. doi:10.1016/j.biocel.2021.106013

Narendra D, Kane LA, Hauser DN, Fearnley IM, Youle RJ. 2010. p62/SQSTM1 is required for Parkininduced mitochondrial clustering but not mitophagy; VDAC1 is dispensable for both. Autophagy 6:1090-1106. doi:10.4161/auto.6.8.13426

North BJ, Marshall BL, Borra MT, Denu JM, Verdin E. 2003. The Human Sir2 Ortholog, SIRT2, Is an NAD+-Dependent Tubulin Deacetylase. Mol Cell 11:437-444. doi:10.1016/s10972765(03)00038-8 
Nowosad A, Creff J, Jeannot P, Culerrier R, Codogno P, Manenti S, Nguyen L, Besson A. 2021. p27 controls autophagic vesicle trafficking in glucose-deprived cells via the regulation of ATAT1mediated microtubule acetylation. Cell Death Dis 12:1-18. doi:10.1038/s41419-021-03759-9

Pagliuso A, Cossart P, Stavru F. 2018. The ever-growing complexity of the mitochondrial fission machinery. Cell Mol Life Sci 75:355-374. doi:10.1007/s00018-017-2603-0

Perdiz D, Lorin S, Leroy-Gori I, Poüs C. 2017. Stress-induced hyperacetylation of microtubule enhances mitochondrial fission and modulates the phosphorylation of Drp1 at 616 Ser. Cell Signalling 39:32-43. doi:10.1016/j.cellsig.2017.07.020

Perdiz D, Mackeh R, Poüs C, Baillet A. 2011. The ins and outs of tubulin acetylation: More than just a post-translational modification? Cell Signalling 23:763-771. doi:10.1016/j.cellsig.2010.10.014

Portran D, Schaedel L, Xu Z, Théry M, Nachury MV. 2017. Tubulin acetylation protects long-lived microtubules against mechanical ageing. Nat Cell Biol 19:391-398. doi:10.1038/ncb3481

Puthalakath H, Huang DCS, O'Reilly LA, King SM, Strasser A. 1999. The Proapoptotic Activity of the $\mathrm{Bcl}-2$ Family Member Bim Is Regulated by Interaction with the Dynein Motor Complex. Mol Cell 3:287-296. doi:10.1016/S1097-2765(00)80456-6

Reed NA, Cai D, Blasius TL, Jih GT, Meyhofer E, Gaertig J, Verhey KJ. 2006. Microtubule Acetylation Promotes Kinesin-1 Binding and Transport. Curr Biol 16:2166-2172. doi:10.1016/i.cub.2006.09.014

Roberts AJ, Kon T, Knight PJ, Sutoh K, Burgess SA. 2013. Functions and mechanics of dynein motor proteins. Nat Rev Mol Cell Biol 14:713-726. doi:10.1038/nrm3667

Shaner NC, Campbell RE, Steinbach PA, Giepmans BNG, Palmer AE, Tsien RY. 2004. Improved monomeric red, orange and yellow fluorescent proteins derived from Discosoma sp. red fluorescent protein. Nat Biotechnol 22:1567-1572. doi:10.1038/nbt1037

Shida T, Cueva JG, Xu Z, Goodman MB, Nachury MV. 2010. The major $\alpha$-tubulin K40 acetyltransferase aTAT1 promotes rapid ciliogenesis and efficient mechanosensation. Proc Natl Acad Sci U S A 107:21517-21522. doi:10.1073/pnas.1013728107

Singh PK, Weber A, Häcker G. 2018. The established and the predicted roles of dynein light chain in the regulation of mitochondrial apoptosis. Cell Cycle 17:1037-1047. doi:10.1080/15384101.2018.1464851

Tang B. 2018. Miro-Working beyond Mitochondria and Microtubules. Cells 7:18-24. doi:10.3390/cells7030018

Vilmen G, Glon D, Siracusano G, Lussignol M, Shao Z, Hernandez E, Perdiz D, Quignon F, Mouna L, Poüs C, Gruffat H, Maréchal V, Esclatine A. 2020. BHRF1, a BCL2 viral homolog, disturbs mitochondrial dynamics and stimulates mitophagy to dampen type I IFN induction. Autophagy 17:1296-1315. doi:10.1080/15548627.2020.1758416

Walker RA, O’Brien E. T, Pryer NK, Soboeiro MF, Voter WA, Erickson HP, Salmon ED. 1988. Dynamic instability of individual microtubules analyzed by video light microscopy: rate constants and transition frequencies. J Cell Biol 107:1437-1448. doi:10.1083/icb.107.4.1437

Wang K, Ma H, Liu H, Ye W, Li Z, Cheng L, Zhang L, Lei Y, Shen L, Zhang F. 2019. The Glycoprotein and Nucleocapsid Protein of Hantaviruses Manipulate Autophagy Flux to Restrain Host Innate Immune Responses. Cell Reports 27:2075-2091.e5. doi:10.1016/j.celrep.2019.04.061

Wang R, Zhu Y, Ren C, Yang S, Tian S, Chen H, Jin M, Zhou H. 2020. Influenza A virus protein PB1-F2 impairs innate immunity by inducing mitophagy. Autophagy 1-16. doi:10.1080/15548627.2020.1725375

West AP, Shadel GS, Ghosh S. 2011. Mitochondria in innate immune responses. Nat Rev Immunol 11:389-402. doi:10.1038/nri2975

Xie R, Nguyen S, McKeehan WL, Liu L. 2010. Acetylated microtubules are required for fusion of autophagosomes with lysosomes. BMC Cell Biol 11:89-100. doi:10.1186/1471-2121-11-89

Yoneyama M, Kikuchi M, Natsukawa T, Shinobu N, Imaizumi T, Miyagishi M, Taira K, Akira S, Fujita T. 2004. The RNA helicase RIG-I has an essential function in double-stranded RNA-induced innate antiviral responses. Nat Immunol 5:730-737. doi:10.1038/ni1087 
Yoneyama M, Suhara W, Fukuhara Y, Sato M, Ozato K, Fujita T. 1996. Autocrine Amplification of Type I Interferon Gene Expression Mediated by Interferon Stimulated Gene Factor 3 (ISGF3). J Biochem 120:160-169. doi:10.1093/oxfordjournals.jbchem.a021379

Yoshizumi T, Ichinohe T, Sasaki O, Otera H, Kawabata S, Mihara K, Koshiba T. 2014. Influenza A virus protein PB1-F2 translocates into mitochondria via Tom40 channels and impairs innate immunity. Nat Commun 5:4713. doi:10.1038/ncomms5713

Zhao C, Zhao W. 2020. NLRP3 Inflammasome-A Key Player in Antiviral Responses. Front Immunol 11. doi:10.3389/fimmu.2020.00211

Zheng K, Jiang Y, He Z, Kitazato K, Wang Y. 2017. Cellular defence or viral assist: the dilemma of HDAC6. J Gen Virol 98:322-337. doi:10.1099/igv.0.000679

Zhong Z, Umemura A, Sanchez-Lopez E, Liang S, Shalapour S, Wong J, He F, Boassa D, Perkins G, Ali SR, McGeough MG, Ellisman MH, Seki E, Gustafsson AB, Hoffman HM, Diaz-Meco MT, Moscat J, Karin M. 2016. NF-KB Restricts inflammasome activation via elimination of damaged mitochondria. Cell 164:896-910. doi:10.1016/i.cell.2015.12.057 
bioRxiv preprint doi: https://doi.org/10.1101/2021.06.11.448012; this version pssted June 11, 2021. The copyright holder for this preprint

A

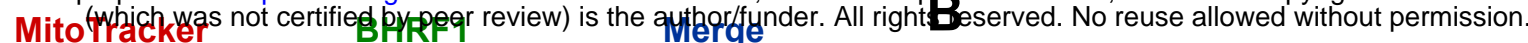
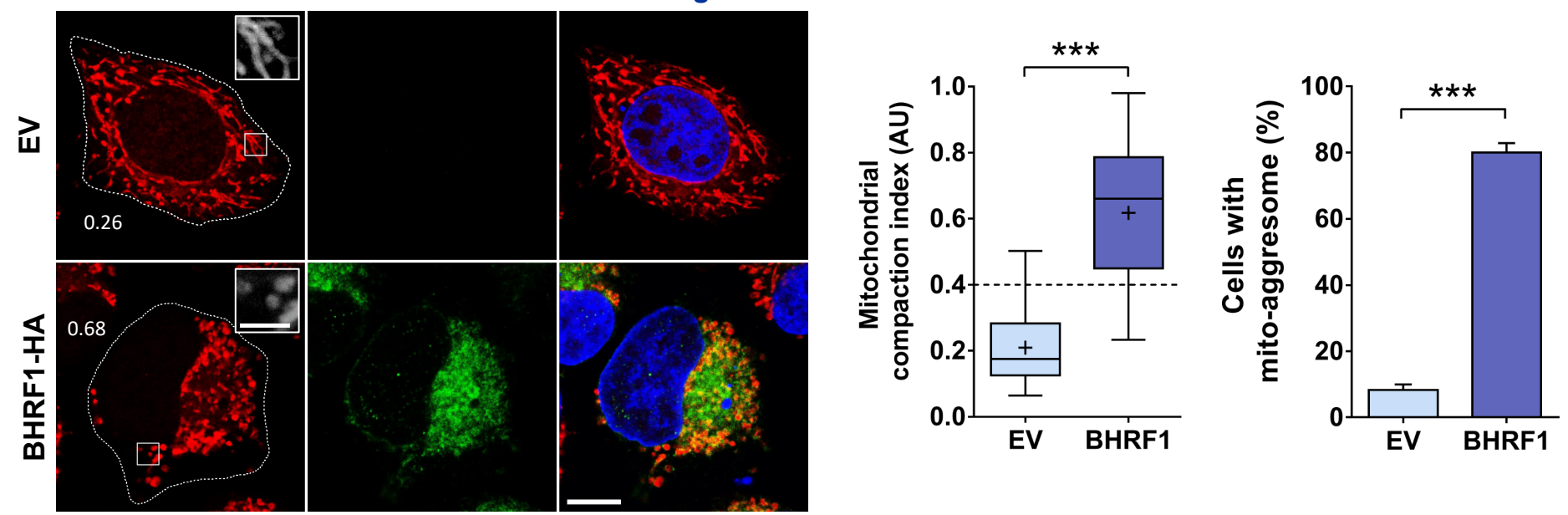

C

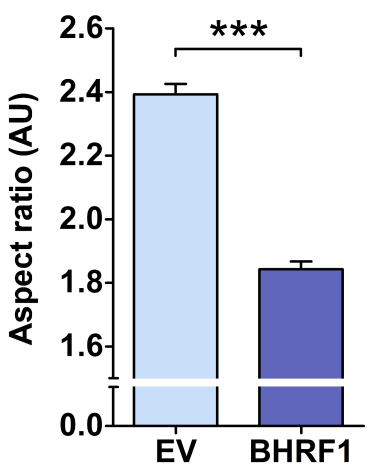

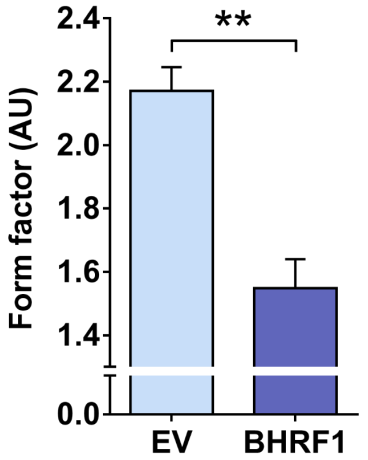

$\mathbf{E}$

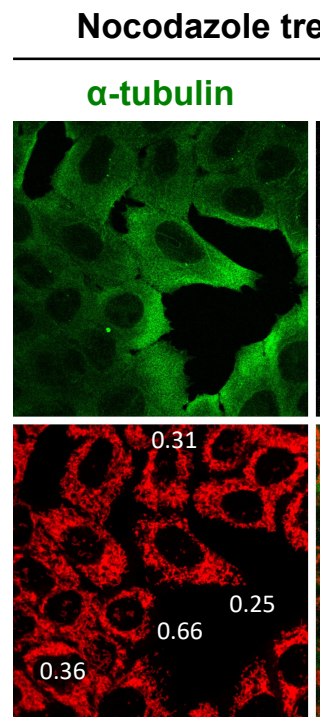

TOM20

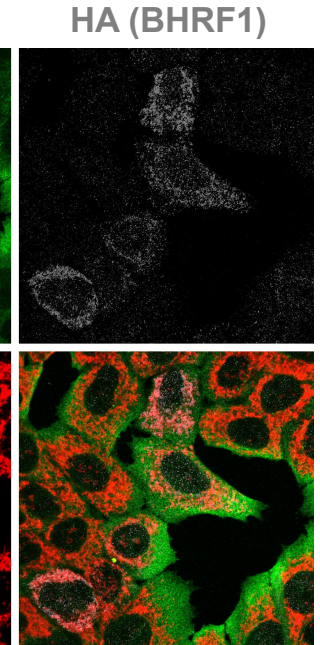

Merge

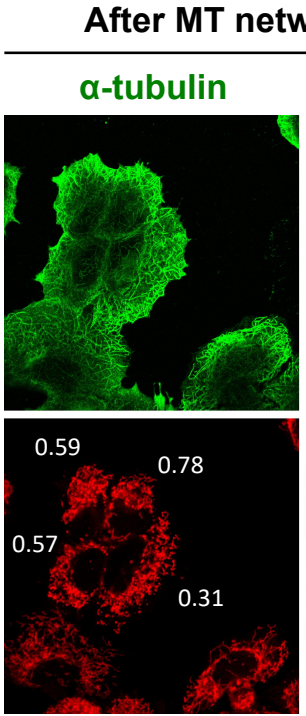

TOM20

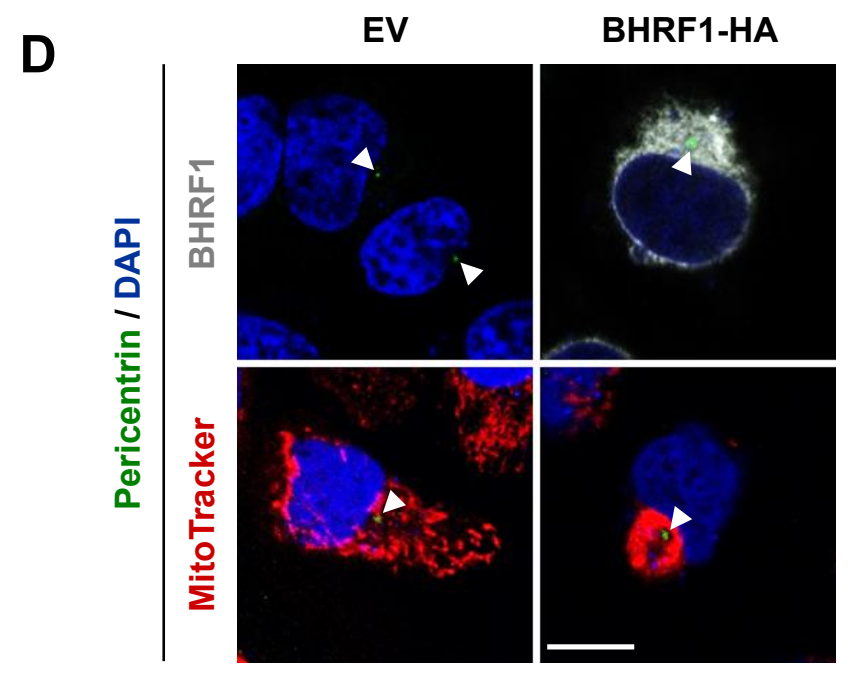

Figure 1. BHRF1 induces the formation of mito-aggresomes in a microtubule dependent manner. (A-C) HeLa cells were transfected for $24 \mathrm{~h}$ with BHRF1-HA plasmid or with EV as a control. Mitochondria were labeled with MitoTracker Red CMXRos and cells immunostained for BHRF1. Nuclei were stained with DAPI. (A) Confocal images with insets (3X) on mitochondrial phenotype. The values of the mitochondrial $\mathrm{Cl}$ are indicated on representative cells. Scale bars: $10 \mu \mathrm{m}$, and $2 \mu \mathrm{m}$ for insets. (B) Left, assessment of mitochondrial aggregation by calculation of Cl. Right, percentage of cells presenting a mito-aggresome $(n=20$ cells). (C) Quantification of mitochondrial fission parameters, aspect ratio (AR; left panel) and form factor (FF, right panel). (D) Confocal images of HeLa cells transfected with BHRF1-HA plasmid (or EV) and immunostained for pericentrin and BHRF1 (upper panel) or mitochondria (lower panel). Nuclei were stained with DAPI. Scale bar: $20 \mu \mathrm{m}$. Arrowheads show the centrosomal localization of pericentrin. (E-F) MT network depolymerization assay. (E) Representative images where BHRF1 expression is visualized by HA immunostaining (gray), the MT network by $\alpha$-tubulin immunostaining (green), and mitochondria are immunostained with an anti-TOM20 antibody (red). The values of the mitochondrial $\mathrm{Cl}$ are indicated on representative cells. Scale bars: $10 \mu \mathrm{m}$, and $2 \mu \mathrm{m}$ for insets. (F) Percentage of BHRF1-HA-positive cells presenting a mito-aggresome $(n=30$ cells $)$ in cells treated with nocodazole and after MT regrowth. Data represent the mean \pm SEM of three independent experiments. ${ }^{* *} \mathrm{P}<0.01 ;{ }^{* *} \mathrm{P}<0.001$ (Student's t-test). 
A bioRxiv preprint doi: https://doi.org/10.1101/2021.06.11.448012; this version posted June 11, 2021. The copyright holder for this preprint
(which was not certified by peer review) is the author/funder.
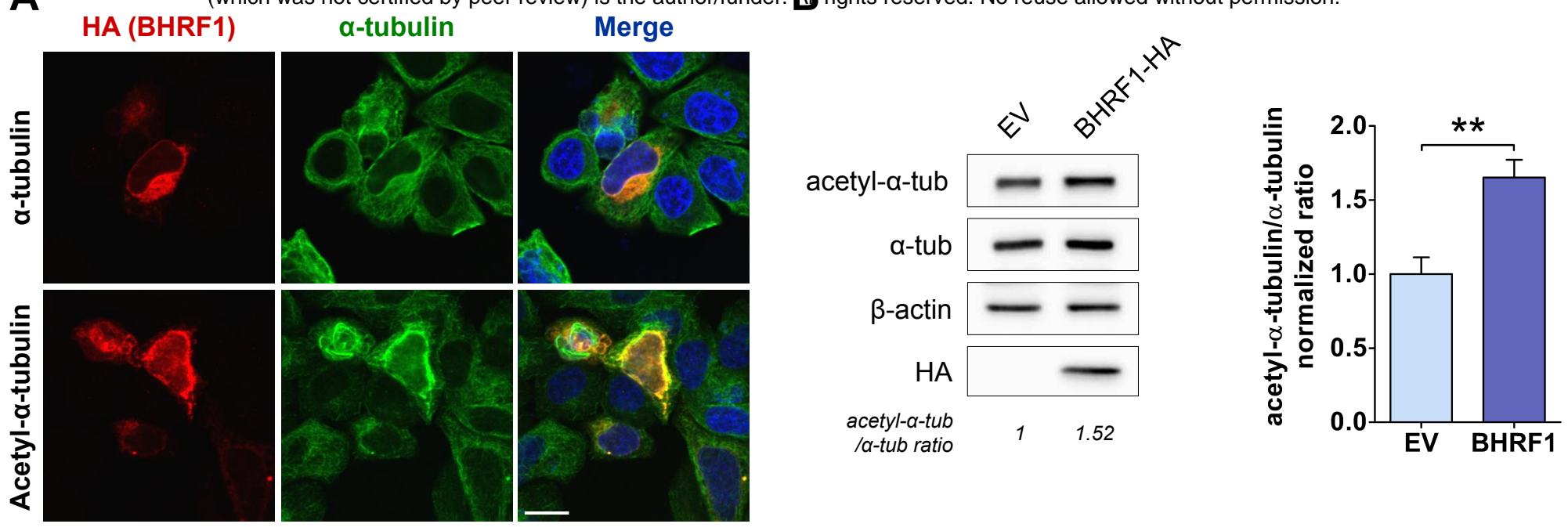

C

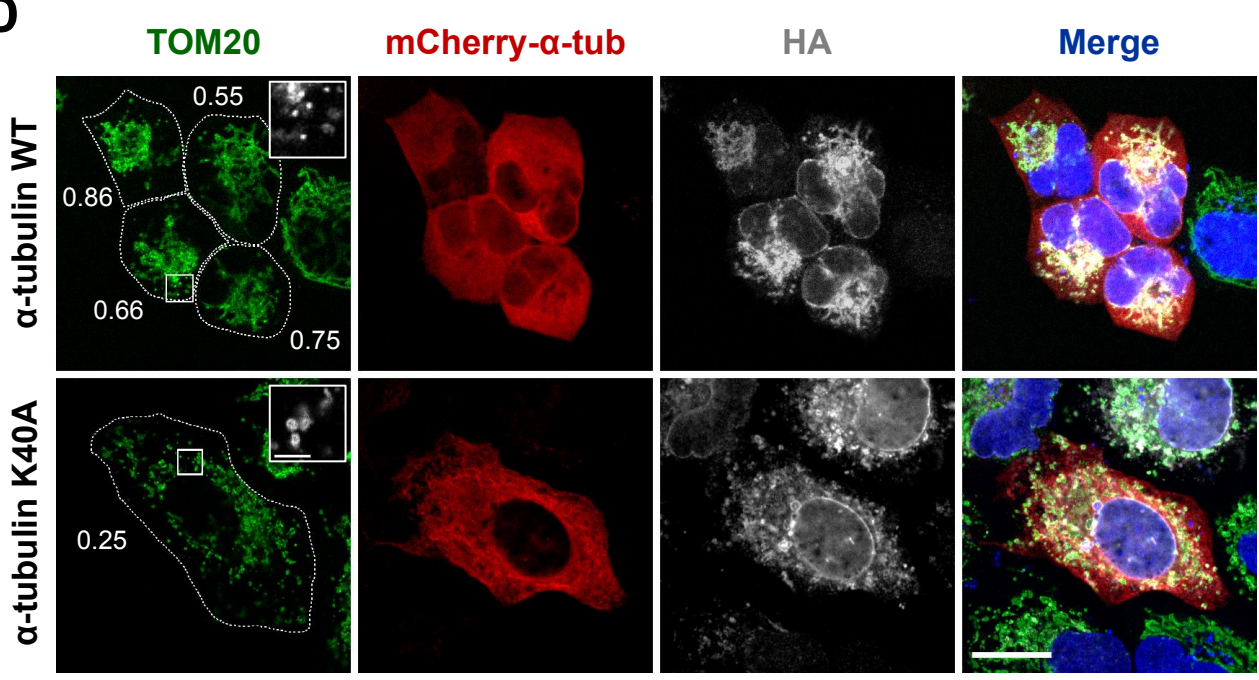

$\mathbf{E}$

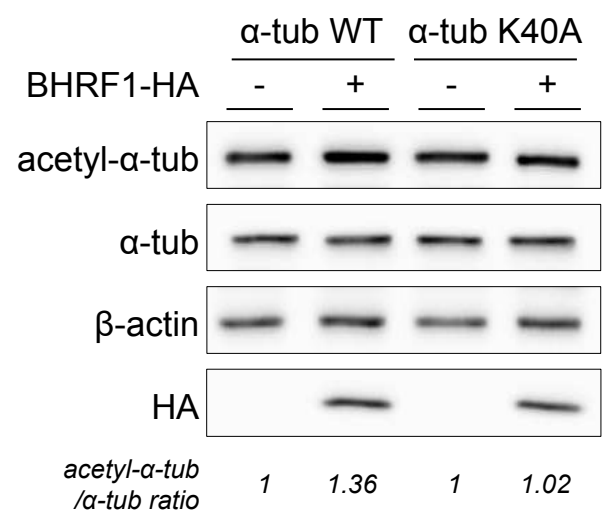

$\mathbf{F}$ $\square \alpha$-tubulin WT $\square \alpha$-tubulin K40A

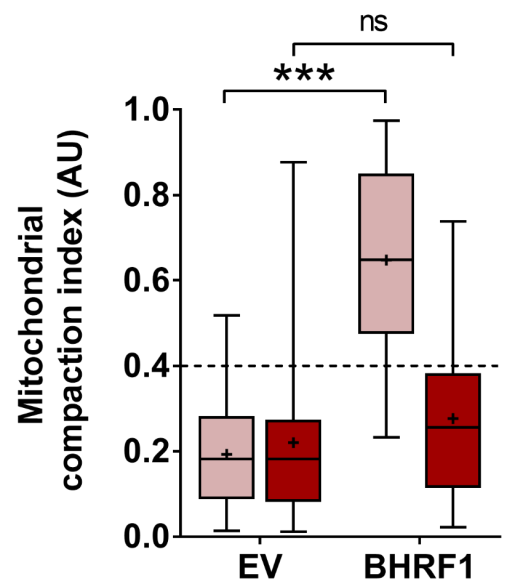

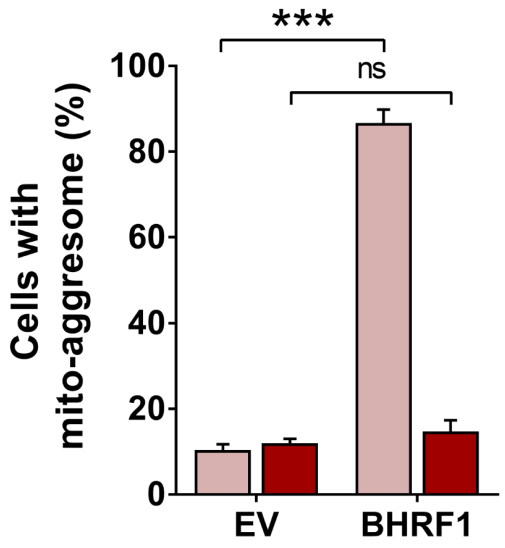

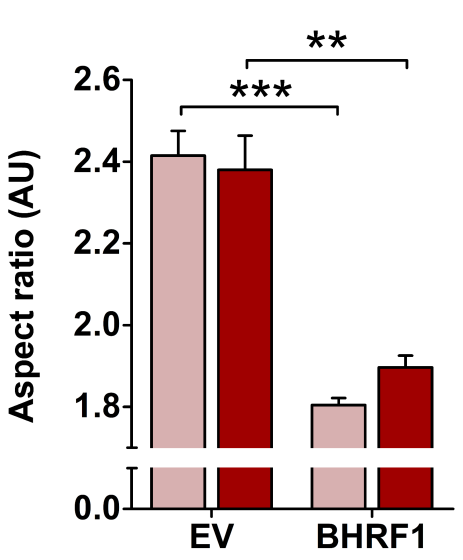

$\alpha$-tubulin WT

$\alpha$-tubulin K40A

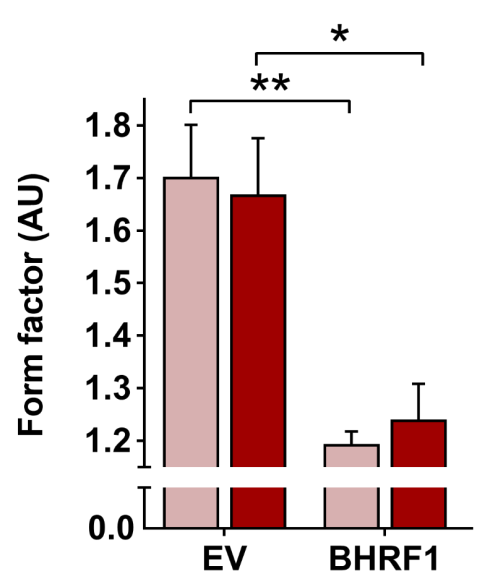

Figure 2. BHRF1 stimulates microtubules hyperacetylation, a process essential for the mito-aggresome formation. (A) Representative images of HeLa cells expressing BHRF1-HA and immunostained for HA and $\alpha$-tubulin (upper panel) or acetyl- $\alpha$-tubulin (lower panel). Nuclei were stained with DAPI. Scale bar: $20 \mu \mathrm{m}$. (B) Left, immunoblot analysis of BHRF1-HA, acetyl and total $\alpha$-tubulin. $\beta$-actin was used as a loading control. Right, the histograms show the normalized ratios of acetyl- $\alpha$-tubulin to total $\alpha$-tubulin. (C-F) HeLa cells were co-transfected with plasmids encoding BHRF1-HA (or EV) and either mCherry- $\alpha$-tubulin WT (K40K) or a non-acetylatable form (K40A). (C) Immunoblot analysis of BHRF1-HA, acetyl and total $\alpha$-tubulin. (D) Confocal images with insets (3X). BHRF1-HA expressing cells were visualized with an anti-HA antibody and mitochondria were immunostained with an anti-TOM20 antibody. Nuclei were stained with DAPI. Scale bars: $10 \mu \mathrm{m}$ and $4 \mu \mathrm{m}$ for insets. Values of mitochondrial $\mathrm{Cl}$ are indicated on representative cells. Images of control cells are presented in Supplementary Figure 3. (E) Quantification of $\mathrm{Cl}$ and cells with mitoaggresomes ( $n=20$ cells). (F) Quantification of mitochondrial fission parameters $(n=20$ cells). Data represent the mean \pm SEM of three independent experiments. $\mathrm{ns}=$ non-significant; $* \mathrm{P}<0.05 ; * * \mathrm{P}<0.01 ; * * \mathrm{P}<0.001$ (Student's t-test). 
bioRxiv preprint doi: https://doi.org/10.1101/2021.06.11.448012; this version posted June 11, 2021. The copyright holder for this preprint (which was not certified by peer r $\mathbf{B e w}$ ) is the author/funder. All rights reserved. Norinse allowed withoutp
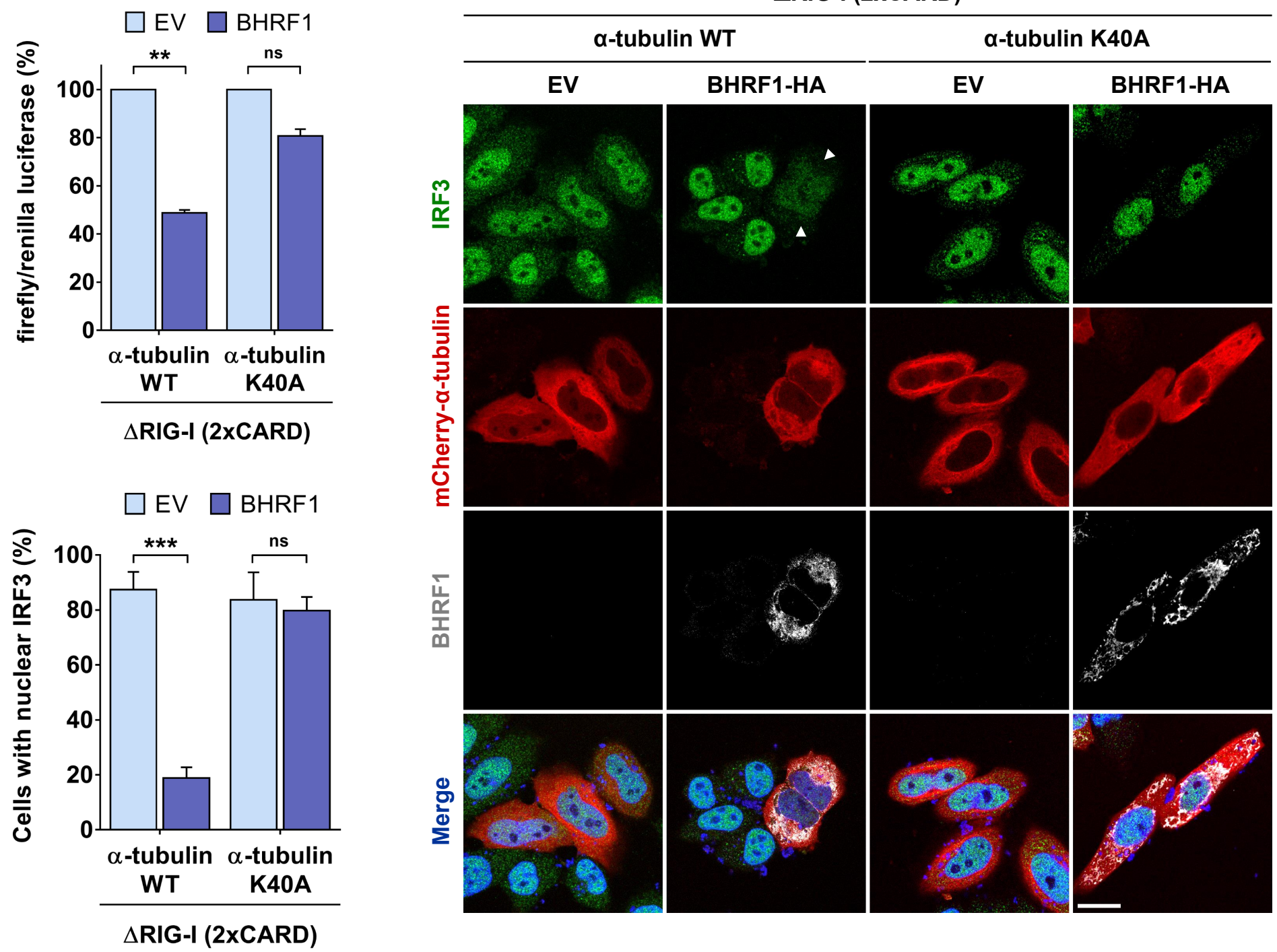

Figure 3. Hyperacetylation of $\alpha$-tubulin is required for BHRF1 to block IFN. (A) Luciferase reporter assay on HEK293T cells co-expressing BHRF1 (or EV) and $\alpha$-tubulin K40A (or WT). Activation of the IFN- $\beta$ promoter was analyzed $24 \mathrm{~h}$ post-transfection. Firefly/renilla luciferase ratios were calculated and normalized to control conditions. (B and C) HeLa cells were co-transfected for $24 \mathrm{~h}$ with plasmids encoding BHRF1-HA (or EV), mCherry- $\alpha$-tubulin K40A (or WT), and $\triangle$ RIG-I (2xCARD). (B) Confocal images. BHRF1-HA-transfected cells were visualized with an anti-HA antibody and cells were immunostained with an-anti IRF3 antibody. Nuclei were stained with DAPI. Scale bar: $20 \mu \mathrm{m}$. Arrowheads indicate BHRF1expressing cells without IRF3 nuclear localization. (C) Percentage of cells expressing mCherry- $\alpha$-tubulin and presenting IRF3 nuclear localization (n $=50$ cells). Data represent the mean \pm SEM of three independent experiments. ns $=$ non-significant; ${ }^{* *} \mathrm{P}<0.01 ;{ }^{* * *} \mathrm{P}<0.001$ (Student's t-test). 
A bioRxiv preprint doi: https://doi.org/10.1101/2021.06.11.448012; this version posted June 11, 2021. The copyright holder for this preprint
(which was not certified by peer review) is the author/funder. All rights reserved. No reuse allowed without permission. Cell lysates SiCTRL

SIATAT1

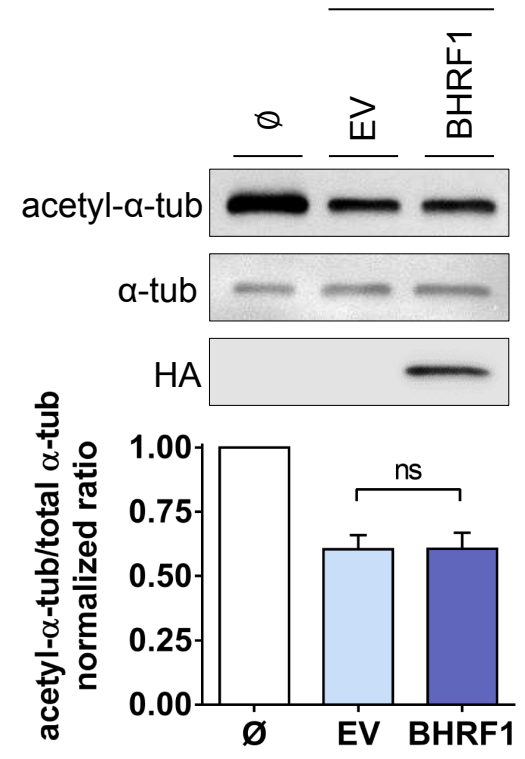

B
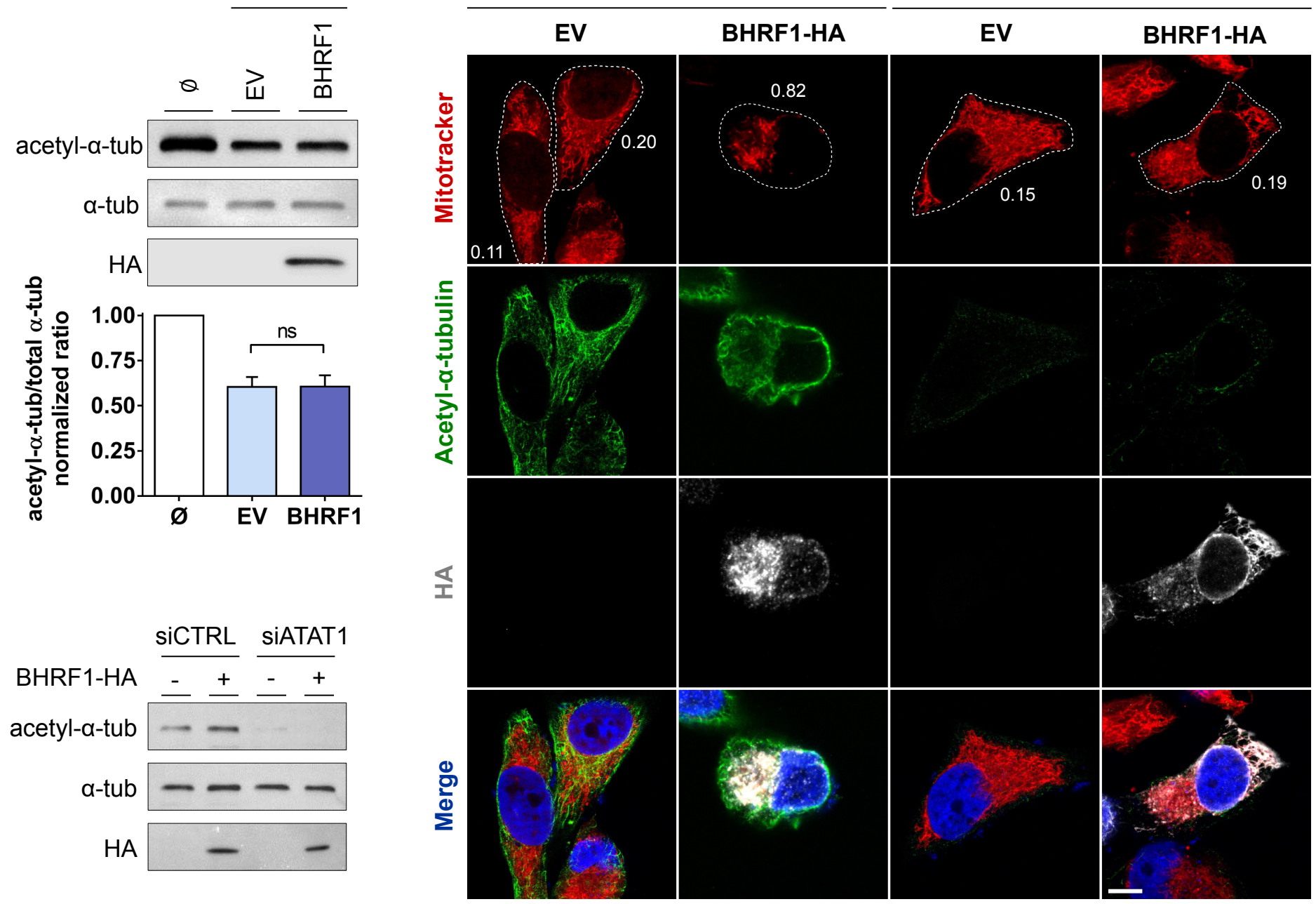

D
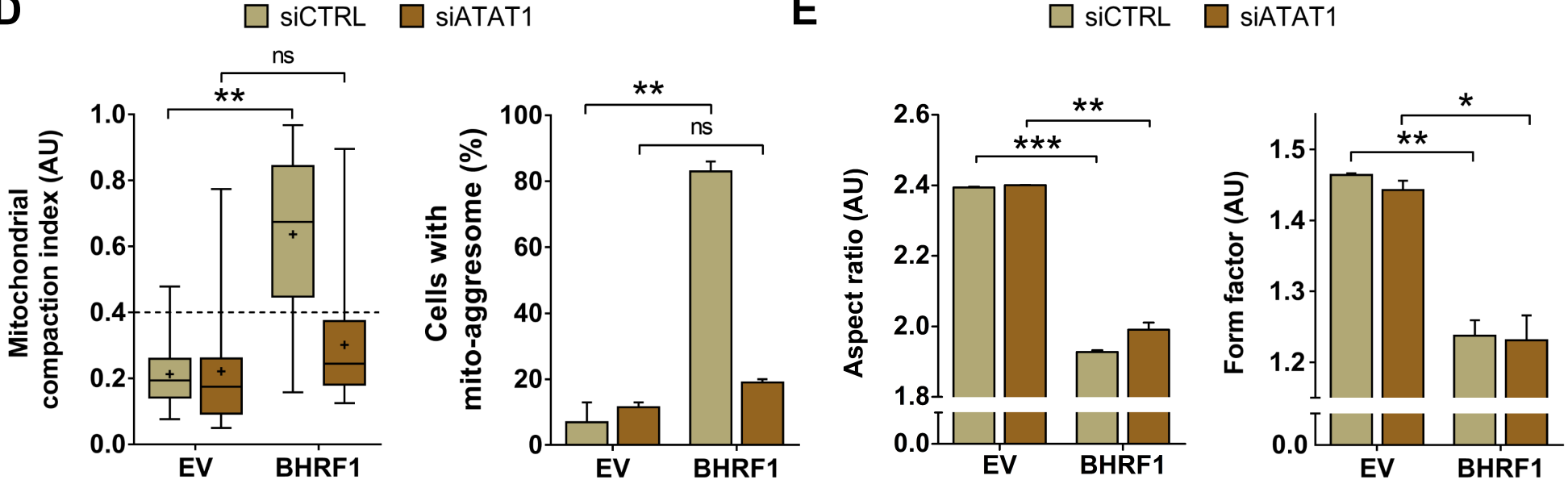

Figure 4. Characterization of MT hyperacetylation mechanism induced by BHRF1. (A) Tubulin deacetylation assay. HeLa cells were transfected with BHRF1-HA plasmid (or EV) and lysed $48 \mathrm{~h}$ post-transfection. Cell lysates were incubated in vitro with porcine brain tubulin and NAD+. BHRF1-HA, acetylated and total $\alpha$-tubulin were detected by immunoblot, and the histogram represents the normalized ratios of acetyl to total $\alpha$-tubulin. (BD) After knockdown of ATAT1, HeLa cells were transfected with BHRF1-HA (or EV) and lysed/fixed $24 \mathrm{~h}$ post-transfection. (B) Extinction of ATAT1 activity visualized by immunoblot analysis of acetylated and total $\alpha$-tubulin. (C) Confocal images. Mitochondria were labeled with MitoTracker CMXRos, and cells were immunostained for acetyl- $\alpha$-tubulin and HA. Nuclei were stained with DAPI. Values of mitochondrial Cl are indicated on representative cells. Scale bar: $20 \mu \mathrm{m}$. (D) Quantification of $\mathrm{Cl}$ and cells with mito-aggresomes ( $\mathrm{n}=20 \mathrm{cells}$ ). (E) Quantification of mitochondrial fission parameters $(n=20$ cells). Data represent the mean \pm SEM of three independent experiments. $n s=n o n-s i g n i f i c a n t ; * P<0.05 ; * * P<0.01$; $* * * \mathrm{P}<0.001$ (Student's t-test). 
bioRxiv preprint doi: https://doi.org/10.1101/2021.06.11.448012; this version posted June 11, 2021. The copyright holder for this preprint (which/Alas not certified by peer review) is the author/funder. All rights reserved. No reuse allowed without permission. MitoTracker myc HA (BHRF1) Merge
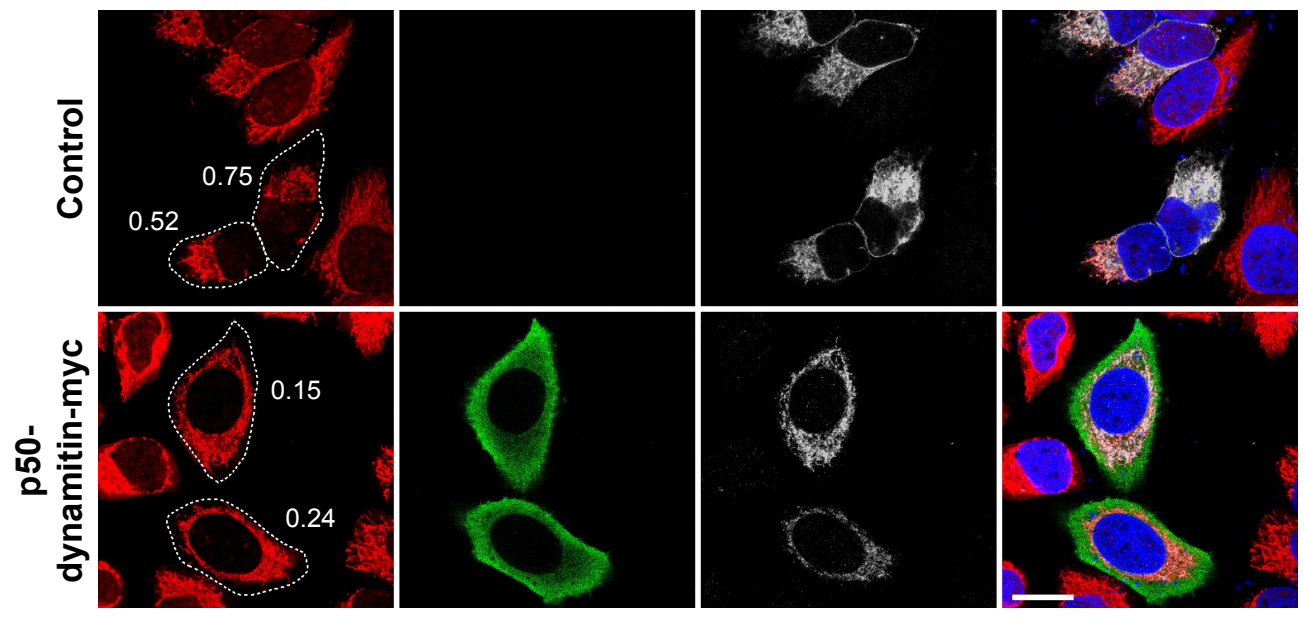

\section{B}

$\square$ Control $\square$ p50-dynamitin

C

Control

p50-dynamitin
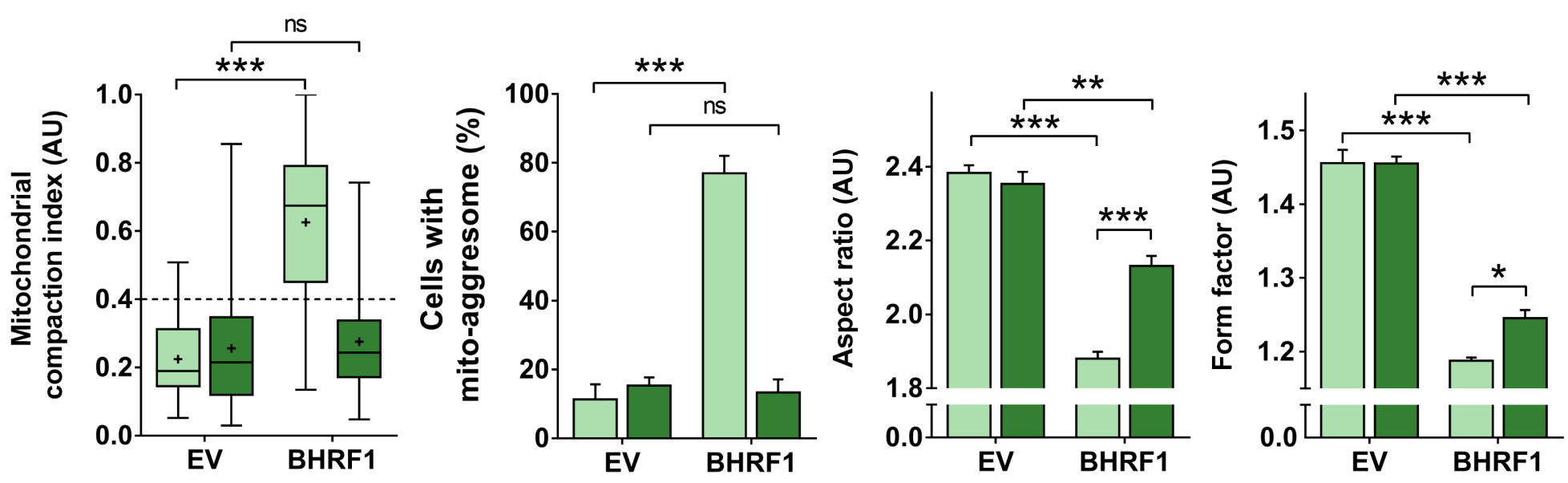

Figure 5. Dynein-based transport is required for BHRF1 to aggregate mitochondria next to the nucleus. (A-C) HeLa cells were co-transfected with plasmids encoding BHRF1-HA (or EV) and p50-dynamitin-myc (or control) for $24 \mathrm{~h}$ and then fixed. (A) Confocal images. Mitochondria were labeled with MitoTracker CMXRos, and cells were immunostained for c-myc and HA. Nuclei were stained with DAPI. Values of mitochondrial Cl are indicated on representative cells. Scale bar: $20 \mu \mathrm{m}$. Images of cells expressing EV and p50-dynamitin-myc are presented in Supplementary Figure 4A. (B) Quantification of $\mathrm{Cl}$ and cells with mito-aggresomes ( $\mathrm{n}=20$ cells). (C) Quantification of mitochondrial fission parameters ( $n=20$ cells). Data represent the mean \pm SEM of three independent experiments. $n s=$ non-significant; $* \mathrm{P}<0.05, * * \mathrm{P}<0.01 ; * * * \mathrm{P}<0.001$ (one-way ANOVA test). 
bioRxiv preprint doi: https://doi.org/10.1101/2021.06.11.448012; this version posted June 11, 2021 The copyright holder for this preprint (which was not certified by peer review) is the author/funder. All rights reserved. No reus Bllowed without permission. a-tubulin K40A

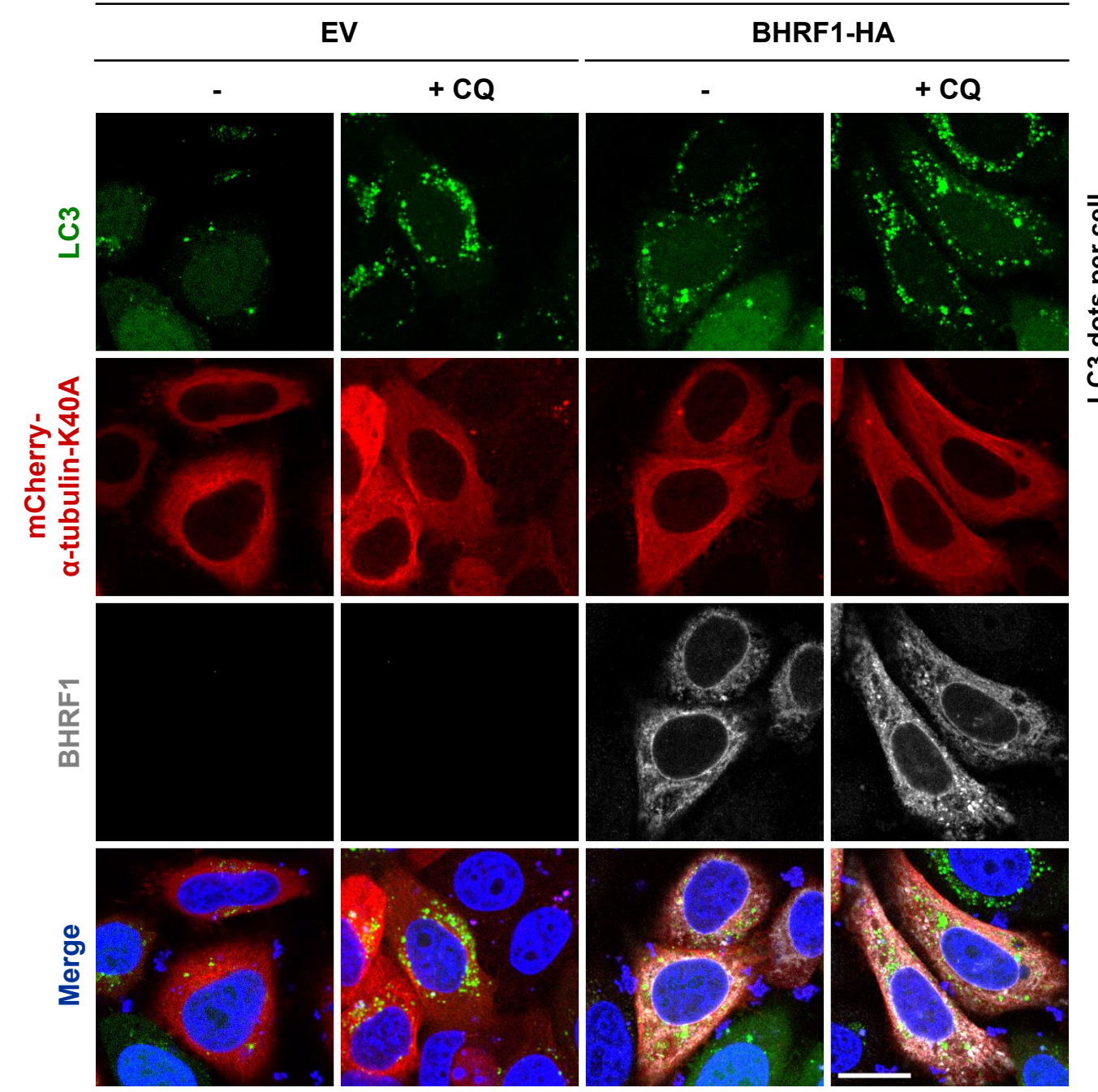

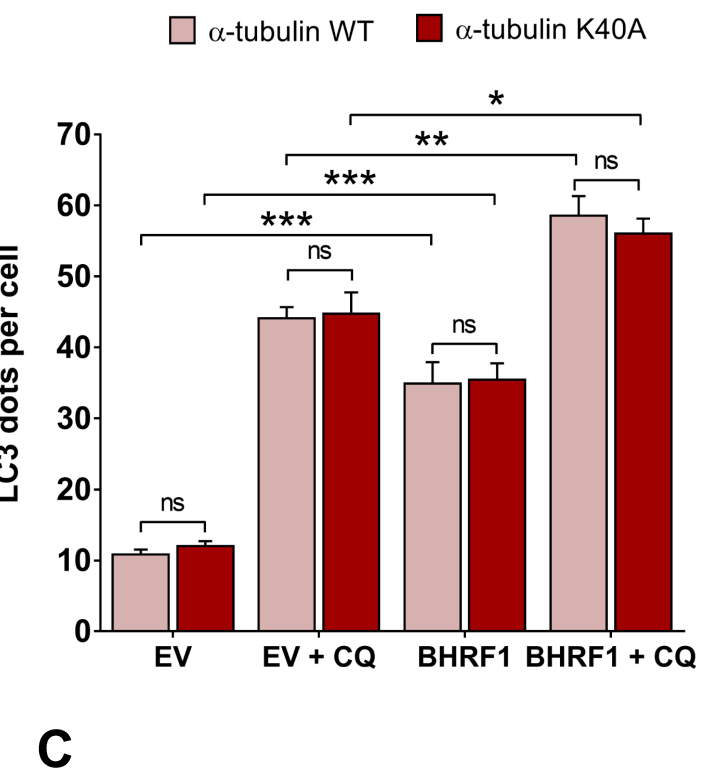

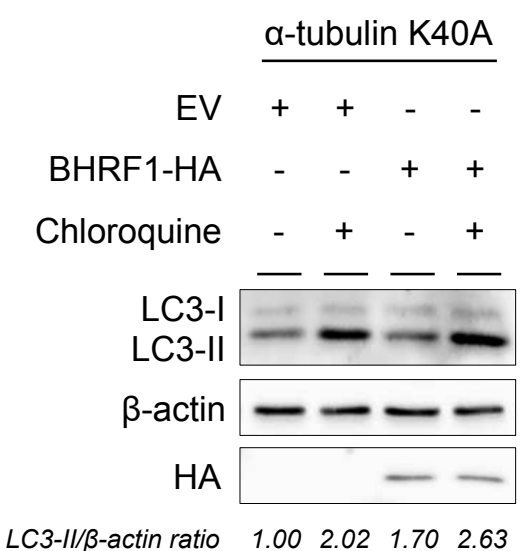

D

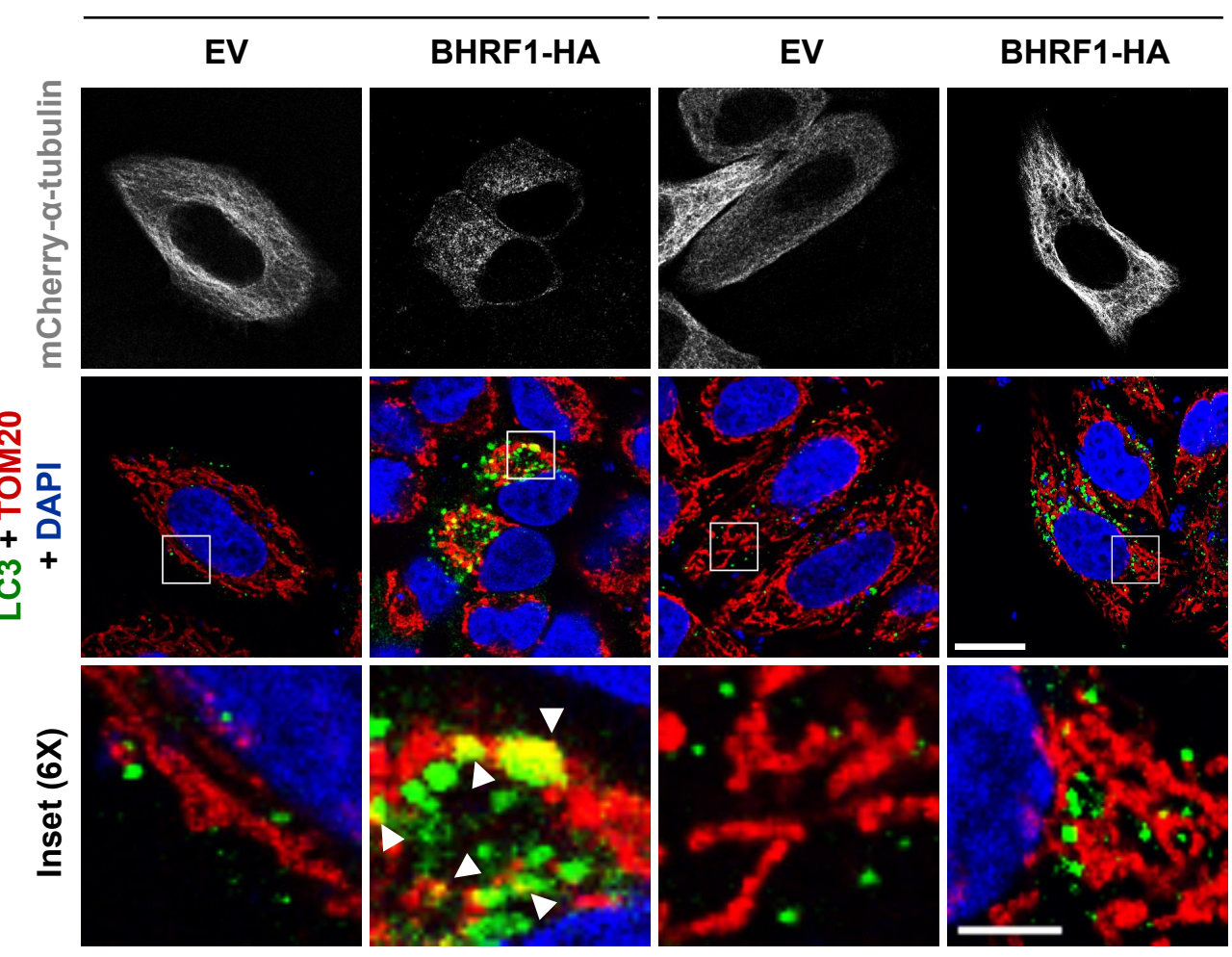

E

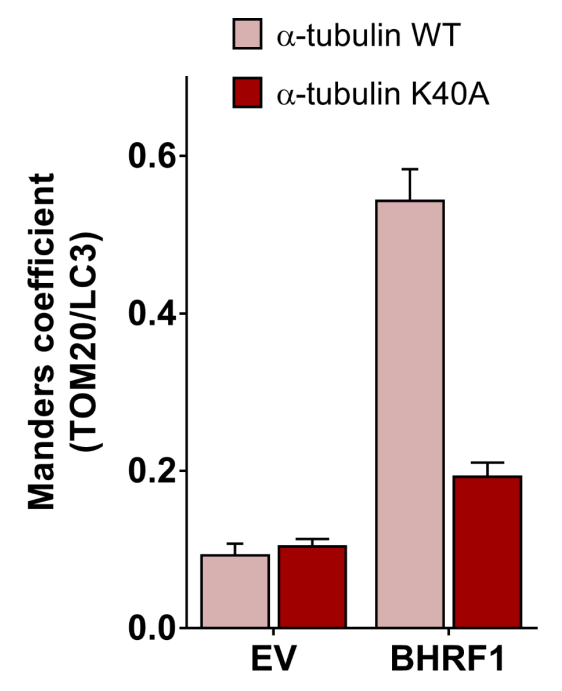

Figure 6. MT hyperacetylation is dispensable for BHRF1-pro-autophagic activity, but essential for mitophagy. (A-C) HeLa cells were co-transfected for $24 \mathrm{~h}$ with plasmids encoding BHRF1-HA (or EV) and mCherry- $\alpha$-tubulin K40A (or WT), and were treated with CQ when indicated. (A) Confocal images. Cells were immunostained for BHRF1 and LC3 and nuclei were stained with DAPI. Scale bar: $10 \mu \mathrm{m}$. Images of cells transfected with mCherry- $\alpha$-tubulin WT are presented in Supplementary Figure 5A. (B) Quantification of LC3 dots in each condition ( $\mathrm{n}=30$ cells). (C) Immunoblot analysis of LC3 expression. (D and E) HeLa cells were co-transfected with BHRF1 (or EV) and mCherry- $\alpha$-tubulin K40A (or WT). Cells were immunostained for TOM20 and LC3, and nuclei stained with DAPI. (D) Confocal images with insets (6X) showing colocalization events (see arrows). Scale bars: $10 \mu \mathrm{m}$ and $5 \mu \mathrm{m}$ for insets. (E) Colocalization level (Manders split coefficient) between mitochondria and autophagosomes. Data represent the mean \pm SEM of three independent experiments. $\mathrm{ns}=$ non-significant; ${ }^{*} \mathrm{P}<0.05 ;{ }^{* *} \mathrm{P}<0.01 ;{ }^{* * *} \mathrm{P}<0.001$ (one-way ANOVA test). 
